\title{
Fermionic observables in the transverse Ising chain
}

\author{
Jakob E. Björnberg ${ }^{\text {a) }}$ \\ Department of Mathematical Sciences, Chalmers University of Technology and the University \\ of Gothenburg, Gothenburg, Sweden
}

(Received 31 August 2016; accepted 14 April 2017; published online 5 May 2017)

\begin{abstract}
We introduce a notion of s-holomorphicity suitable for certain quantum spin systems in one dimension and define two observables in the critical transverse-field Ising model which have this property. The observables are defined using graphical representations in the complex plane and are analogous to Smirnov's FK-Ising and spin-Ising observables, respectively. We also briefly discuss scaling limits of these observables. Published by AIP Publishing. [http://dx.doi.org/10.1063/1.4982637]
\end{abstract}

\section{INTRODUCTION}

Recent years have seen tremendous progress on the understanding of planar models in statistical physics, particularly the (classical) Ising model at criticality. A major breakthrough in this area was the proof of convergence, to conformally covariant scaling limits, of fermionic observables in the critical Ising model, first on the square lattice by Smirnov, ${ }^{23,24}$ and later on all isoradial graphs by Chelkak and Smirnov. ${ }^{12}$

The fermionic observables enjoy a crucial property called s-holomorphicity, a strong form of discrete analyticity. Besides satisfying a discrete version of the Cauchy-Riemann relations, if a function $F_{\delta}$ is s-holomorphic, then one may define a discrete primitive $H_{\delta}=\operatorname{Im}\left(\int^{\delta} F_{\delta}^{2}\right)$ of its square. Moreover this function $H_{\delta}$ is very close to being (discrete) harmonic. When combined with control of the behaviour of $H_{\delta}$ at the boundary of the domain, this allows to deduce convergence of the fermionic observables from convergence of solutions to discrete boundary-value problems.

The identification of the scaling limits of these and related observables has subsequently led to some outstanding results on the critical planar Ising model, settling several predictions from conformal field theory. This includes convergence of the energy-density, ${ }^{16}$ correlation functions, ${ }^{11}$ as well as interfaces to SLE-curves ${ }^{10}$ and loops to CLE-processes, ${ }^{4,18}$ to mention but a few.

In this note, we start to consider similar questions in the context of one-dimensional quantum spin-systems, specifically the transverse-field (quantum) Ising model, hereafter abbreviated TFIM. $^{21}$ This model has Hamiltonian given by

$$
-\mathcal{H}_{N}=J \sum_{x=1}^{N-1} \sigma_{x}^{(3)} \sigma_{x+1}^{(3)}+h \sum_{x=1}^{N} \sigma_{x}^{(1)}, \text { acting on } \otimes_{x=1}^{N} \mathbb{C}^{2},
$$

where $\sigma^{(3)}=\left(\begin{array}{cc}1 & 0 \\ 0 & -1\end{array}\right)$ and $\sigma^{(1)}=\left(\begin{array}{ll}0 & 1 \\ 1 & 0\end{array}\right)$ are the spin- $\frac{1}{2}$ Pauli matrices, and $J, h>0$ give the couplingand transverse-field-strengths, respectively. (For $h=0$, this is just the classical Ising model.) We will be working with the ground-state (zero temperature), where the model is known to undergo a phase-transition as the ratio $h / J$ is varied, at the critical point $h / J=1 .^{22}$ The phase-transition is continuous. $^{8}$

It is well-known that the тFIм in $d$ dimensions possesses a graphical, probabilistic representation in $\mathbb{Z}^{d} \times \mathbb{R}$, and it behaves in many ways like a classical Ising model in $d+1$ dimensions, see, e.g., the results in Refs. 6 and 7. One may thus ask whether the results mentioned above, on conformal invariance in the two-dimensional classical Ising model at criticality, have analogs in the one-dimensional quantum model.

\footnotetext{
a)E-mail: jakob.bjornberg@gmail.com.
} 
This note is a first step in this direction. We introduce a notion of s-holomorphicity for functions on $\mathbb{Z}+i \mathbb{R} \subseteq \mathbb{C}$; we show that functions that satisfy this enjoy (analogs of) the key properties that hold in the classical case; and we define two observables in the critical TFIM which we show to be s-holomorphic.

The two observables are defined using two different graphical representations, and in analogy with their classical counterparts, we call them the FK- and spin-observable, respectively. The graphical representations that we consider may be obtained as limits of classical counterparts on $\mathbb{Z}+i(\varepsilon \mathbb{Z})$ as $\varepsilon \rightarrow 0$. The latter graphs are all isoradial, and some of the key quantities we work with can be interpreted as limits of the corresponding quantities for isoradial graphs. ${ }^{12}$ We give examples of this in Section IV B. However, for all our definitions and results, we work directly in the "continuous" setting $\mathbb{Z}+i \mathbb{R}$ and the rescaled version $\delta \mathbb{Z}+i \mathbb{R}$. Our results for the tFIM hold for the choice of parameters $h=J=\frac{1}{2 \delta}$.

We do not go into the details for scaling limits (as $\delta \rightarrow 0$ ) of our observables here, but we expect this to be very similar to the classical case. As we discuss in Section VI, we expect analogous reasoning and estimates to show that our observables converge to the same scaling-limits as their classical counterparts. We also expect that these and related observables can be used to determine the scaling limits of physically interesting quantities. In the classical case, variants of the spin-observable have been used to obtain scaling limits of correlation functions, ${ }^{11,16}$ and we expect that similar results can be obtained for the TFIM.

\section{A. Outline and main contributions}

After reviewing the graphical representations of the TFIM in Section II, we give our definition of sholomorphicity in Section III and prove some key properties of s-holomorphic functions in Proposition 3.2. We introduce and study our two fermionic observables in Sections IV and V, respectively. The main results are that these observables satisfy our definition of s-holomorphicity, stated precisely in Theorems 4.2 and 5.2.

\section{B. Bibliographical remark}

Shortly after this paper was made public, $\mathrm{Li}^{20}$ announced a complete proof of convergence of the FK-observable considered here, as well as the FK-interface to $\mathrm{SLE}_{16 / 3}$, in the scaling limit. Li independently arrived at equivalent definitions of the FK-observable and s-holomorphicity as presented here and supplied the details necessary to prove convergence. He does not consider the spin-observable. Most likely his results are useful for proving convergence of that observable as well.

\section{GRAPHICAL REPRESENTATIONS OF THE TFIM}

We briefly review three graphical representations of the TFIM. They may be obtained using a Lie-Trotter expansion, see, e.g., Refs. 2, 5, 8, and 17 for details. We also present a version of the Kramers-Wannier duality; as for the classical case, this allows us to easily identify the critical parameters of the model (but for rigorous proofs see Refs. 22 and 8).

We write the partition function $\mathcal{Z}_{N, \beta}=\mathcal{Z}_{N, \beta}(h, J)=\operatorname{tr}\left(e^{-\beta \mathcal{H}_{N}}\right)$, where $\mathcal{H}_{N}$ is the Hamiltonian (1), and $\beta>0$ is the inverse-temperature. For illustration we will also consider the two-point correlation

$$
\left\langle\sigma_{x}^{(3)} \sigma_{y}^{(3)}\right\rangle_{N, \beta}=\operatorname{tr}\left(\sigma_{x}^{(3)} \sigma_{y}^{(3)} e^{-\beta \mathcal{H}_{N}}\right) / \mathcal{Z}_{N, \beta} .
$$

Thermodynamic limits are obtained for $N \rightarrow \infty$, and the ground-state is obtained by also letting $\beta \rightarrow \infty$.

The TFIM on $\{1, \ldots, N\}$ maps onto stochastic models in the rectangular domain $\Omega=[1, N]+$ $i[0, \beta] \subseteq \mathbb{C}$. We write

$$
\Omega^{\bullet}=\{1, \ldots, N\}+i[0, \beta], \quad \Omega^{\circ}=(1 / 2+\{1, \ldots, N-1\})+i[0, \beta] .
$$

We will let $\xi^{\bullet}$ and $\xi^{\circ}$ denote independent Poisson processes on $\Omega^{\bullet}$ and $\Omega^{\circ}$, respectively. Their respective rates will be denoted $r^{\bullet}$ and $r^{\circ}$ and will be the functions of $h$ and $J$. We write $\mathbb{E}_{r^{\bullet}, r^{\circ}}[\cdot]$ for the law (expectation operator) governing them, and $\xi=\xi^{\bullet} \cup \xi^{\circ}$. Elements of $\xi^{\bullet}$ will be represented 

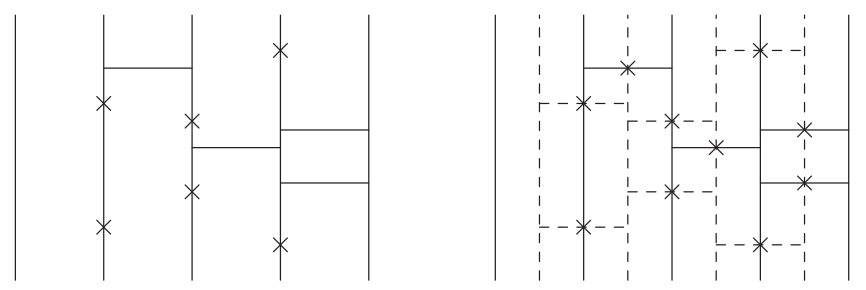

FIG. 1. Left: Illustration of the FK-representation. Cuts $(\times)$ disconnect, bridges (horizontal line segments) connect, and top and bottom of the intervals are identified. The number $k(\xi)$ of components is 5. Right: The same FK-sample $\xi$ (solid) with its dual $\xi^{\prime}$ (dashed).

graphically by $\times$ and called "cuts"; an element $(x+1 / 2)+i t$ of $\xi^{\circ}$ will be represented as a horizontal line-segment between $x+i t$ and $(x+1)+i t$ and called a "bridge." The interpretation of these objects will differ slightly for the three different representations, as we now describe. See Figures 1 and 2, for example.

\section{A. FK-representation}

For this representation, we set $r^{\bullet}=h$ and $r^{\circ}=2 \mathrm{~J}$. We interpret the cuts $x+i t \in \xi^{\bullet}$ as severing a line-segment $x+i[0, \beta]$, and the bridges $\xi^{\circ}$ as connecting neighbouring line segments. Thus the configuration $\xi$ is a partly continuous percolation-configuration. The maximal connected subsets of $\Omega^{\bullet}$ are called components, and their number is denoted $k^{\bullet}(\xi)$. The components may be defined with respect to various boundary conditions, but for now we only consider the "vertically periodic" boundary condition, meaning that the points at the top and bottom of $\Omega^{\bullet}$ are identified (i.e., we treat $[0, \beta]$ as a circle). See Figure 1.

The FK-representation expresses

$$
\mathcal{Z}_{N, \beta}=e^{\beta J(N-1)} \mathbb{E}_{h, 2 J}\left[2^{k^{\bullet}(\xi)}\right], \quad\left\langle\sigma_{x}^{(3)} \sigma_{y}^{(3)}\right\rangle_{N, \beta}=\frac{\mathbb{E}_{h, 2 J}\left[\mathbb{I}\{x \leftrightarrow y\} 2^{k^{\bullet(\xi)}}\right]}{\mathbb{E}_{h, 2 J}\left[2^{k^{\bullet(\xi)}}\right]},
$$

where $\{x \leftrightarrow y\}$ denotes the event that $x, y \in\{1, \ldots, N\}$ belong to the same connected component.

With an FK-configuration $\xi$, we can associate a dual configuration $\xi^{\prime}$, whose connected components are subsets of $\Omega^{\circ}$ rather than $\Omega^{\bullet}$. For simplicity, we describe this in the case when $\xi^{\bullet}$ has no cuts on the left- or rightmost intervals $1+i[0, \beta]$ and $N+i[0, \beta]$. We obtain $\xi^{\prime}$ by drawing a bridge from $(x-1 / 2)+i t$ to $(x+1 / 2)+i t$ for each cut $x+i t \in \xi^{\bullet}$, and placing a cut $\times$ at $(x+1 / 2)+i t$ whenever $\xi^{\circ}$ has a bridge there. See Figure 1. Objects, such as cuts, bridges, and components, pertaining to $\xi^{\prime}$ will be referred to as dual and those of $\xi$ as primal when a distinction needs to be made. The number of dual components will be denoted $k^{\circ}(\xi)$. It turns out that $\xi^{\prime}$ also has the law of a FK-configuration,
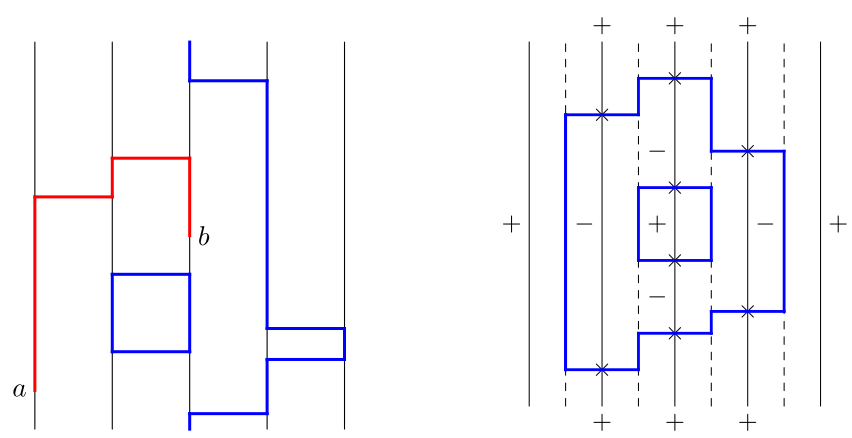

FIG. 2. Left: Sample of the random-parity representation with source set $A=\{a, b\}$. Intervals where $\psi=1$ are drawn bold, with red for the unique path between $a$ and $b$ and blue for the loops. Right: Duality between the space-time spin and randomparity representations. Values + and - indicate the value of $\sigma(z)$ on the corresponding interval in $\Omega^{\bullet}$, and these values flip at cuts $\times$. Blue vertical intervals mark where $\psi(z)=1$. 
with adjusted parameters. We will return to this construction when we define the FK-observable in Section IV.

\section{B. Random-parity representation}

For this representation, we set $r^{\bullet}=0$ and $r^{\circ}=J$, thus there are only bridges. We use auxiliary configurations $\psi \in\{0,1\}^{N}$ together with a fixed, finite subset $A \subseteq \Omega^{\bullet}$ of sources. The configuration $\psi$ is extended to a function $\psi_{A}: \Omega^{\bullet} \rightarrow\{0,1\}$, in such a way that it depends on $\xi^{\circ}$ and $A$, using the following rules. The function $\psi_{A}(x+i t)$ is equal to $\psi(x)$ for $t$ from 0 to the first time of either a bridge $(x \pm 1 / 2)+i t \in \xi^{\circ}$ or a source $x+i t \in A$. At such a point, it switches to $1-\psi(x)$. Then it stays at that value until it encounters another bridge-endpoint or source, where it switches back to $\psi(x)$, and so on. See Figure 2 for an example.

The subset of $\Omega^{\bullet}$ where $\psi_{A}$ takes value 1 is denoted $I\left(\psi_{A}\right)=\psi_{A}^{-1}(1)$ and will for definiteness be taken to be closed. We denote its total length $\left|I\left(\psi_{A}\right)\right|$. We will only be considering the cases when either $A=\varnothing$ or $A$ consists of two points; in the former case $I\left(\psi_{A}\right)$ may be viewed as a collection of loops and in the latter case loops plus a unique path connecting the two points of $A$.

We impose the periodicity constraint that $\psi(x+i \beta)=\psi(x)$ for all $x \in\{1, \ldots, N\}$; if $x \in A$, then the correct interpretation is $\psi(x+i \beta)=1-\psi(x)$ due to the switching-rule. Hence we discount some configurations $\xi$, specifically those where some line $x+i[0, \beta]$ meets an odd number of switchingpoints. As we will see presently, this discounting can be done formally by redefining $\left|I\left(\psi_{A}\right)\right|=\infty$ when the constraint is violated.

The random-parity representation expresses

$$
\begin{aligned}
& \mathcal{Z}_{N, \beta}=e^{\beta h N+\beta J(N-1)} \mathbb{E}_{0, J}\left[\sum_{\psi \in\{0,1\}^{N}} \exp \left(-2 h\left|I\left(\psi_{\emptyset}\right)\right|\right)\right], \\
& \left\langle\sigma_{x}^{(3)} \sigma_{y}^{(3)}\right\rangle_{N, \beta}=\frac{\mathbb{E}_{0, J}\left[\sum_{\psi \in\{0,1\}^{N}} \exp \left(-2 h\left|I\left(\psi_{\{x, y\}}\right)\right|\right)\right]}{\mathbb{E}_{0, J}\left[\sum_{\psi \in\{0,1\}^{N}} \exp \left(-2 h\left|I\left(\psi_{\emptyset}\right)\right|\right)\right]} .
\end{aligned}
$$

This representation is a quantum version of Aizenman's random-current representation. ${ }^{1}$ There is a notion of planar duality also for this representation, mapping onto the space-time spin representation, which we describe now.

\section{Space-time spin representation}

This representation plays a less prominent role in this note and is mainly interesting since it is dual to the random-parity representation. We now set $r^{\bullet}=h$ and $r^{\circ}=0$, thus there are only cuts. We let $\Sigma(\xi)$ denote the set of functions $\sigma: \Omega^{\bullet} \rightarrow\{-1,+1\}$ which are constant between points of $\xi^{\bullet}$, change values at the points of $\xi^{\bullet}$, and satisfy the periodicity constraint $\sigma(x)=\sigma(x+i \beta)$ for all $x \in\{1, \ldots, N\}$. See Figure 2. (For definiteness, we may take $\sigma^{-1}(+1)$ to be closed; also note that for some $\xi$, we have $\Sigma(\xi)=\varnothing$.)

For readability, we also write $\sigma_{x}(t)$ for $\sigma(x+i t)$. The space-time spin representation expresses

$$
\begin{aligned}
& \mathcal{Z}_{N, \beta}=e^{\beta h N} \mathbb{E}_{h, 0}\left[\sum_{\sigma \in \Sigma(\xi)} \exp \left(J \sum_{z=1}^{N-1} \int_{0}^{\beta} \sigma_{z}(t) \sigma_{z+1}(t) d t\right)\right], \\
& \left\langle\sigma_{x}^{(3)} \sigma_{y}^{(3)}\right\rangle_{N, \beta}=\frac{\mathbb{E}_{h, 0}\left[\sum_{\sigma \in \Sigma(\xi)} \sigma(x) \sigma(y) \exp \left(J \sum_{z=1}^{N-1} \int_{0}^{\beta} \sigma_{z}(t) \sigma_{z+1}(t) d t\right)\right]}{\mathbb{E}_{h, 0}\left[\sum_{\sigma \in \Sigma(\xi)} \exp \left(J \sum_{z=1}^{N-1} \int_{0}^{\beta} \sigma_{z}(t) \sigma_{z+1}(t) d t\right)\right]} .
\end{aligned}
$$

\section{Kramers-Wannier duality}

We now describe a duality between the random-parity and spin-representations. We will associate (in a reversible way) a spin-configuration $\sigma: \Omega^{\bullet} \rightarrow\{-1,+1\}$ with a "dual" random-parityconfiguration $\psi=\psi_{\varnothing}: \Omega^{\circ} \rightarrow\{0,1\}$. Note that the domain of $\psi$ is $\Omega^{\circ}$ rather than $\Omega^{\circ}$. We impose 
the "wired" boundary condition

$$
\begin{aligned}
\sigma(1+i t)=\sigma(N+i t)=\sigma(x) & =\sigma(x+i \beta)=+1, \\
& \text { for all } t \in[0, \beta], x \in\{1, \ldots, N\} .
\end{aligned}
$$

As we will see, this will automatically lead to the boundary condition,

$$
\psi(x+1 / 2)=\psi((x+1 / 2)+i \beta)=0, \text { for all } x \in\{1, \ldots, N\} .
$$

Subject to the boundary conditions, the sums over $\sigma$ in (4) and $\psi$ in (3) contribute with at most one nonzero term each, hence they will not be written out.

We construct $\psi$ from $\sigma$ as follows, see Figure 2. If two neighbouring points $x+i t$ and $(x+1)$ $+i t$ have the same spin-value, $\sigma(x+i t)=\sigma((x+1)+i t)$, then we set $\psi((x+1 / 2)+i t)=0$; otherwise if $\sigma(x+i t) \neq \sigma((x+1)+i t)$, then we set $\psi((x+1 / 2)+i t)=1$. If $x+i t \in \xi^{\bullet}$ is a point of spin-flip for $\sigma$, we draw a bridge between $(x-1 / 2)+i t$ and $(x+1 / 2)+i t$. Thus the bridges form a Poisson process of rate $h$.

Writing $\mathcal{Z}_{N, \beta}^{+}(h, J)$ for the partition function (4) associated with the spin-configurations, we have that

$$
\begin{aligned}
\mathcal{Z}_{N, \beta}^{+}(h, J) & =e^{\beta h N} \mathbb{E}_{h, 0}\left[\exp \left(J \sum_{x=1}^{N-1} \int_{0}^{\beta} \sigma_{x}(t) \sigma_{x+1}(t) d t\right)\right] \\
& =e^{\beta h N} \mathbb{E}_{h, 0}\left[\exp \left(J \sum_{x=1}^{N-1} \int_{0}^{\beta}[1-2 \psi((x+1 / 2)+i t)] d t\right)\right] \\
& =e^{\beta h N+\beta J(N-1)} \mathbb{E}_{h, 0}[\exp (-2 J|I(\psi)|)] .
\end{aligned}
$$

Comparing with (3), we see that the last factor

$$
\mathbb{E}_{h, 0}[\exp (-2 J|I(\psi)|)]=e^{-\beta J(N-1)-\beta h(N-2)} \mathcal{Z}_{N-1, \beta}^{0}(J, h),
$$

where $\mathcal{Z}_{N-1, \beta}^{0}(J, h)$ is the partition function associated with the $\psi$ 's with the prescribed boundary condition. Note that the order of the parameters $h, J$ is swapped.

We conclude that

$$
\mathcal{Z}_{N, \beta}^{+}(h, J)=e^{2 \beta h} \mathcal{Z}_{N-1, \beta}^{0}(J, h)
$$

Assuming (as can be justified) the existence of the limit as well as its independence of the boundary condition, we deduce that the free energy $f(h, J)=\lim _{N, \beta \rightarrow \infty} \frac{1}{\beta N} \log \mathcal{Z}_{N, \beta}(h, J)$ satisfies $f(h, J)=f(J$, $h$ ). This symmetry is consistent with a phase-transition at $h=J$. In the rest of this note, we consider only the critical case, $h=J$.

\section{S-HOLOMORPHIC FUNCTIONS}

\section{A. Discrete domains}

As indicated above, we will be considering functions on (bounded subsets of) $\delta \mathbb{Z}+i \mathbb{R} \subseteq \mathbb{C}$. We use the notation

$$
\mathbb{C}_{\delta}^{\bullet}=\delta \mathbb{Z}+i \mathbb{R}, \quad \mathbb{C}_{\delta}^{\circ}=\mathbb{C}_{\delta}^{\bullet}+\delta / 2, \quad \text { and } \quad \mathbb{C}_{\delta}^{\diamond}=\left(\mathbb{C}_{\delta}^{\bullet} \cup \mathbb{C}_{\delta}^{\circ}\right)+\delta / 4 .
$$

We will sometimes refer to points of $\mathbb{C}_{\delta}^{\bullet}$ as primal or black, points of $\mathbb{C}_{\delta}^{\circ}$ as dual or white, and points of $\mathbb{C}_{\delta}^{\diamond}$ as medial. See Figure 3 for illustrations of the definitions that follow.

Let $\partial_{\delta}:[0,1] \rightarrow \mathbb{C}$ be a simple closed rectangular path, consisting of vertical and horizontal line segments, whose vertical segments are restricted to $\mathbb{C}_{\delta}^{\bullet}$. Let $\Omega_{\delta}$ denote the bounded component of $\mathbb{C} \backslash \partial_{\delta}[0,1]$. Such a domain $\Omega_{\delta}$ will be referred to as a primal (discrete) domain. We also write, for $* \in\{\bullet, \circ\}$,

$$
\Omega_{\delta}^{*}=\overline{\Omega_{\delta}} \cap \mathbb{C}_{\delta}^{*}, \quad \partial \Omega_{\delta}^{*}=\Omega_{\delta}^{*} \cap \partial \Omega_{\delta}, \quad \Omega_{\delta}^{* \text { int }}=\Omega_{\delta}^{*} \backslash \partial \Omega_{\delta}^{*} .
$$

Note that $\Omega_{\delta}^{*}$ consists of a collection of vertical line segments and $\partial \Omega_{\delta}^{*}$ of vertical line segments together with a finite number of points (forming the horizontal part of the boundary). We similarly define a dual (discrete) domain $\Omega_{\delta}$ by shifting the above definition by $\delta / 2$ (thus swapping $\mathbb{C}_{\delta}^{\bullet}$ and $\left.\mathbb{C}_{\delta}^{\circ}\right)$. 

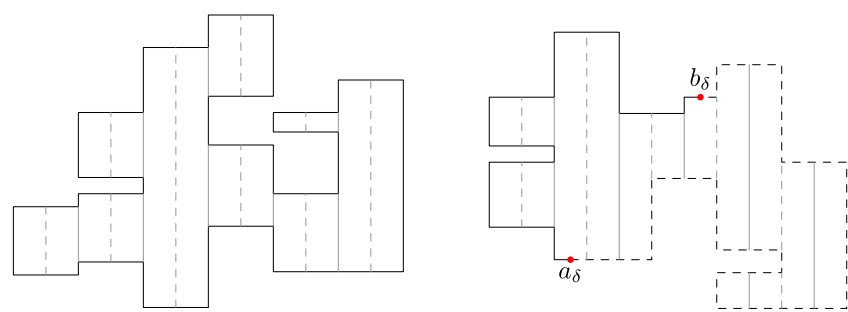

FIG. 3. Left: A primal domain $\Omega_{\delta}$. The boundary is drawn with solid black lines, while $\Omega_{\delta}^{\bullet}$ consists of the solid black and gray vertical lines and $\Omega_{\delta}^{\circ}$ of the dashed gray vertical lines. Right: A Dobrushin domain $\Omega_{\delta}$ with $\partial_{\delta}^{\bullet}$ drawn solid and $\partial_{\delta}^{\circ}$ dashed.

We will also consider Dobrushin domains. For this we let $a_{\delta}, b_{\delta} \in \mathbb{C}_{\delta}^{\diamond}$ be two distinct medial points, and let $\partial_{\delta}:[0,1] \rightarrow \mathbb{C}$ be a simple closed positively oriented rectangular path, satisfying

$$
\partial_{\delta}(0)=\partial_{\delta}(1)=a_{\delta}, \quad \partial_{\delta}(1 / 2)=b_{\delta} .
$$

We define $\partial_{\delta}^{\bullet}, \partial_{\delta}^{\circ}:[0,1] \rightarrow \mathbb{C}$ by

$$
\partial_{\delta}^{\circ}(t)=\partial_{\delta}(t / 2), \quad \partial_{\delta}^{\bullet}(t)=\partial_{\delta}(1-t / 2), \quad t \in[0,1] .
$$

Thus $\partial_{\delta}^{\circ}$ goes from $a_{\delta}$ to $b_{\delta}$ in the counter-clockwise direction, and $\partial_{\delta}^{\bullet}$ goes from $a_{\delta}$ to $b_{\delta}$ in the clockwise direction. We require that $a_{\delta}$ is placed so that the first point of $\mathbb{C}_{\delta}^{\bullet} \cup \mathbb{C}_{\delta}^{\circ}$ visited by $\partial_{\delta}^{\bullet}$ (as it travels clockwise from $a_{\delta}$ to $b_{\delta}$ ) belongs to $\mathbb{C}_{\delta}^{\bullet}$; this will be convenient later. Finally we assume that the vertical segments of $\partial_{\delta}^{\bullet}$ and $\partial_{\delta}^{\circ}$ belong to $\mathbb{C}_{\delta}^{\bullet}$ and $\mathbb{C}_{\delta}^{\circ}$, respectively. Again we write $\Omega_{\delta}$ for the bounded component of $\mathbb{C} \backslash \partial_{\delta}[0,1]$, and we refer to the triple $\left(\Omega_{\delta}, a_{\delta}, b_{\delta}\right)$ as a discrete Dobrushin domain. We define $\Omega_{\delta}^{\bullet}, \partial \Omega_{\delta}^{\bullet}, \Omega_{\delta}^{\bullet, \text { int }}$, as well as $\Omega_{\delta}^{\circ}, \partial \Omega_{\delta}^{\circ}$, $\Omega_{\delta}^{\circ \text {,int }}$, as in (5).

For a primal, dual, or Dobrushin domain $\Omega_{\delta}$, and $* \in\{\bullet, \circ\}$, we define the vertical and horizontal parts of the boundary $\partial \Omega_{\delta}^{*}$ by

$$
\begin{aligned}
& \partial^{\mathrm{v}} \Omega_{\delta}^{*}=\overline{\left\{z \in \partial \Omega_{\delta}^{*}: z+\varepsilon \notin \Omega_{\delta} \text { or } z-\varepsilon \notin \Omega_{\delta} \text { for small enough } \varepsilon>0\right\}}, \\
& \partial^{\mathrm{h}} \Omega_{\delta}^{*}=\left\{z \in \partial \Omega_{\delta}^{*}: z+i \varepsilon \notin \Omega_{\delta} \text { or } z-i \varepsilon \notin \Omega_{\delta} \text { for small enough } \varepsilon>0\right\} .
\end{aligned}
$$

We also let $\partial^{\mathrm{v}} \Omega_{\delta}=\partial^{\mathrm{v}} \Omega_{\delta}^{\bullet} \cup \partial^{\mathrm{v}} \Omega_{\delta}^{\circ}$ and $\partial^{\mathrm{h}} \Omega_{\delta}=\partial^{\mathrm{h}} \Omega_{\delta}^{\bullet} \cup \partial^{\mathrm{h}} \Omega_{\delta}^{\circ}$. In other words, $\partial^{\mathrm{v}} \Omega_{\delta}$ consists of the vertical segments of $\partial \Omega_{\delta}$, and $\partial^{\mathrm{h}} \Omega_{\delta}$ of the endpoints of segments in $\overline{\Omega_{\delta}}$. We finally make the assumption on $\Omega_{\delta}$ that if $z \in \partial^{\mathrm{v}} \Omega_{\delta}$ then at least one of $z \pm \delta / 2$ belongs to the interior $\Omega_{\delta}^{\bullet \text {,int }} \cup \Omega_{\delta}^{\text {o,int }}$.

In what follows, we will consider triples $\left(\Omega_{\delta}, a_{\delta}, b_{\delta}\right)$ which are either discrete Dobrushin domains, alternatively discrete primal or dual domains with two marked points $a_{\delta}, b_{\delta} \in \partial \Omega_{\delta}$. One may think of these as approximating a simply connected domain $\Omega \subseteq \mathbb{C}$ with two marked points $a, b$ on its boundary.

\section{B. S-holomorphic functions}

Let $\Omega_{\delta}$ be a discrete domain, as above, and $F: \Omega_{\delta} \rightarrow \mathbb{C}$ a function. We will be using the notation

$$
\dot{F}(z):=\lim _{\varepsilon \rightarrow 0} \frac{F(z+i \varepsilon)-F(z)}{\varepsilon}, \quad \text { with } \varepsilon \in \mathbb{R},
$$

for the derivative of $F$ in the "vertical" direction, when it exists. We similarly write $\ddot{F}(z)$ for the second derivative.

For a complex number $\zeta$, with $|\zeta|=1$, and $z \in \mathbb{C}$, we write

$$
\operatorname{Proj}[z ; \zeta]=\operatorname{Proj}[z ; \zeta \mathbb{R}]=\frac{1}{2}\left(z+\bar{z} \zeta^{2}\right)
$$

for the projection of $z$ onto (the straight line through 0 ) $\zeta$. The cases when $\zeta=e^{ \pm i \pi / 4}$ will be particularly important in what follows, and we will write $\ell(\uparrow)=e^{-i \pi / 4} \mathbb{R}$ and $\ell(\downarrow)=e^{i \pi / 4} \mathbb{R}$. (This choice of notation will be motivated below, in the context of the FK-observable.) We define

$$
F^{\uparrow}(z)=\operatorname{Proj}[F(z) ; \ell(\uparrow)], \quad F^{\downarrow}(z)=\operatorname{Proj}[F(z) ; \ell(\downarrow)] .
$$

Note that $F(z)=F^{\uparrow}(z)+F^{\downarrow}(z)$ since $\ell(\uparrow) \perp \ell(\downarrow)$ (Figure 4). 

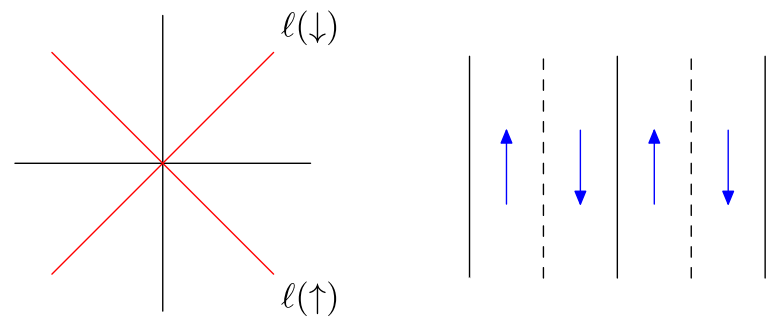

FIG. 4. Left: The lines $\ell(\uparrow)$ and $\ell(\downarrow)$. Right: Illustration of conditions (9) and (10) in Definition 3.1. For a pair of adjacent black and white points, separated by an arrow in direction $\alpha \in\{\uparrow, \downarrow\}$, the projections of $F$ onto $\ell(\alpha)$ are the same.

Definition 3.1 (s-holomorphicity). A function $F: \Omega_{\delta}^{\bullet} \cup \Omega_{\delta}^{\circ} \rightarrow \mathbb{C}$ is s-holomorphic at a point $z \in \Omega_{\delta}^{\circ \text {,int }} \cup \Omega_{\delta}^{\bullet, \text { int }}$ if the following hold:

$$
\frac{1}{\delta}(F(z+\delta / 2)-F(z-\delta / 2))+i \dot{F}(z)=0
$$

and

$$
F^{\uparrow}(z)=F^{\uparrow}(z-\delta / 2), \quad F^{\downarrow}(z)=F^{\downarrow}(z+\delta / 2), \quad \text { if } z \in \Omega_{\delta}^{\circ \text { int }},
$$

respectively,

$$
F^{\uparrow}(z)=F^{\uparrow}(z+\delta / 2), \quad F^{\downarrow}(z)=F^{\downarrow}(z-\delta / 2), \quad \text { if } z \in \Omega_{\delta}^{\bullet, \text { int }} .
$$

If $F$ is s-holomorphic at every point $z \in \Omega_{\delta}^{\bullet, \text { int }} \cup \Omega_{\delta}^{\circ \text {,int }}$, then we simply say that $F$ is s-holomorphic in $\Omega_{\delta}$.

The choice of the term s-holomorphic is mainly motivated by Proposition 3.2, which is completely analogous to the classical case (e.g., Proposition 3.6 of Ref. 12).

Note that (8) is equivalent to the two equations,

$$
\begin{aligned}
\dot{F}^{\uparrow}(z) & =\frac{i}{\delta}\left(F^{\downarrow}(z+\delta / 2)-F^{\downarrow}(z-\delta / 2)\right), \text { and } \\
\dot{F}^{\downarrow}(w) & =\frac{i}{\delta}\left(F^{\uparrow}(z+\delta / 2)-F^{\uparrow}(z-\delta / 2)\right) .
\end{aligned}
$$

In establishing s-holomorphicity, we will check (11) as well as (9) and (10).

As for the classical case, the main benefit of s-holomorphic functions $F$ is that they have wellbehaved discrete analogs of $\operatorname{Im}\left(\int F^{2}\right)$. In the next result, we write $\Delta_{\delta}$ for the appropriate Laplacian operator given by

$$
\left[\Delta_{\delta} f\right](z)=\ddot{f}(z)+\frac{1}{\delta^{2}}(f(z+\delta)+f(z-\delta)-2 f(z)) .
$$

We say that a function $h$ is $\Delta_{\delta}$-harmonic (respectively, $\Delta_{\delta}$-sub- or $\Delta_{\delta}$-super-harmonic) at a point $z \in \mathbb{C}_{\delta}^{\bullet} \cup \mathbb{C}_{\delta}^{\circ}$ if $\left[\Delta_{\delta} h\right](z)=0$ (respectively, $\left[\Delta_{\delta} h\right](z) \geq 0$ or $\left.\left[\Delta_{\delta} h\right](z) \leq 0\right)$.

Proposition 3.2. Let $F$ be s-holomorphic in $\Omega_{\delta}$. Then there is a function $H: \Omega_{\delta}^{\bullet} \cup \Omega_{\delta}^{\circ} \rightarrow \mathbb{R}$, unique up to an additive constant, satisfying the following. First, for $z$ such that $[z, z+\delta / 2] \subseteq \Omega_{\delta}$,

$$
H(z+\delta / 2)-H(z)=\left\{\begin{array}{l}
+\left|F^{\downarrow}(z)\right|^{2}, \text { if } z \in \Omega_{\delta}^{\circ}, \\
-\left|F^{\uparrow}(z)\right|^{2}, \text { if } z \in \Omega_{\delta}^{\bullet},
\end{array}\right.
$$

and second, for any $z \in \Omega_{\delta}^{\bullet} \cup \Omega_{\delta}^{\circ}$,

$$
\dot{H}(z)=\frac{2}{\delta} F^{\uparrow}(z) F^{\downarrow}(z) .
$$

Moreover, we have for all $u \in \Omega_{\delta}^{\bullet, \text { int }}$ and $w \in \Omega_{\delta}^{\circ \text {,int }}$ that

$$
\left[\Delta_{\delta} H\right](u)=|\dot{F}(u)|^{2} \quad \text { and } \quad\left[\Delta_{\delta} H\right](w)=-|\dot{F}(w)|^{2} .
$$

Hence $H$ is $\Delta_{\delta}$-sub-harmonic in $\Omega_{\delta}^{\bullet, \text { int }}$ and $\Delta_{\delta}$-super-harmonic in $\Omega_{\delta}^{\circ \text {,int }}$. 
Remark 3.3. The function $H(z)$ is a discrete analog of $\frac{1}{\delta} \operatorname{Im}\left(\int^{z} F^{2}\right)$. Indeed, since $2 F^{\uparrow}(z) F^{\downarrow}(z)$ $=\operatorname{Re}\left[F(z)^{2}\right]$, we see that if $u, u^{\prime} \in \Omega_{\delta}^{\bullet}$ with $u=x+i y$ and $u^{\prime}=x+i y^{\prime}$ for some $x \in \delta \mathbb{Z}$ and $y, y^{\prime} \in \mathbb{R}$ such that $\left[u, u^{\prime}\right] \subseteq \Omega_{\delta}^{*}$, then

$$
\begin{aligned}
H\left(u^{\prime}\right)-H(u) & =\int_{y}^{y^{\prime}} \dot{H}(x+i t) d t=\frac{1}{\delta} \int_{y}^{y^{\prime}} \operatorname{Re}\left[F(x+i t)^{2}\right] d t \\
& =\frac{1}{\delta} \int_{y}^{y^{\prime}} \operatorname{Im}\left[i F(x+i t)^{2}\right] d t=\frac{1}{\delta} \operatorname{Im}\left[\int_{u}^{u^{\prime}} F(z)^{2} d z\right] .
\end{aligned}
$$

Similarly, if $v=u+\delta \in \Omega_{\delta}^{\bullet}$ and $w=u+\delta / 2=v-\delta / 2$ are midway between $u$ and $v$, then, using $F^{\uparrow}(z)^{2}+F^{\downarrow}(z)^{2}=i \operatorname{Im}\left[F(z)^{2}\right]$, we have

$$
\begin{aligned}
H(v)-H(u) & =H(w+\delta / 2)-H(w)+H(w)-H(w-\delta / 2) \\
& =\left|F^{\downarrow}(w)\right|^{2}-\left|F^{\uparrow}(w)\right|^{2}=\frac{1}{i}\left(F^{\uparrow}(w)^{2}+F^{\downarrow}(w)^{2}\right) \\
& =\operatorname{Im}\left[F(w)^{2}\right] .
\end{aligned}
$$

Proof of Proposition 3.2. Uniqueness up to an additive constant follows since if we fix $H(u)$ for some point $u$, then for $v \neq u$, we may obtain the value $H(v)$ by integrating using (13) and (14). To see that $H$ is well-defined, consider a situation such as in Figure 5. It suffices to show that the total increment of $H$ around the blue (left) contour and around the green (right) contour are both equal to 0 . We prove this for the green (right) contour, the other one being similar.

Let us write, for $a, b, c, t_{1}, t_{2} \in \mathbb{R}$ and $j=1,2, u_{j}=a+i t_{j}, w_{j}=b+i t_{j}$, and $v_{j}=c+i t_{j}$. We have

$$
\begin{aligned}
{\left[H\left(v_{2}\right)-\right.} & \left.H\left(v_{1}\right)\right]+\left[H\left(w_{1}\right)-H\left(w_{2}\right)\right]=\int_{t_{1}}^{t_{2}}(\dot{H}(c+i t)-\dot{H}(b+i t)) d t \\
& =\frac{2}{\delta} \int_{t_{1}}^{t_{2}} F^{\downarrow}(b+i t)\left(F^{\uparrow}(c+i t)-F^{\uparrow}(b+i t)\right) d t \\
& =\frac{1}{i} \int_{t_{1}}^{t_{2}} 2 F^{\downarrow}(b+i t) \dot{F}^{\downarrow}(b+i t) d t \\
& =\frac{1}{i}\left(F^{\downarrow}\left(w_{2}\right)^{2}-F^{\downarrow}\left(w_{1}\right)^{2}\right)=\left|F^{\downarrow}\left(w_{2}\right)\right|^{2}-\left|F^{\downarrow}\left(w_{1}\right)\right|^{2} \\
& =\left[H\left(v_{2}\right)-H\left(w_{2}\right)\right]-\left[H\left(v_{1}\right)-H\left(w_{1}\right)\right] .
\end{aligned}
$$

That is, the increments around the green contour satisfy

$$
\left[H\left(v_{2}\right)-H\left(v_{1}\right)\right]+\left[H\left(w_{2}\right)-H\left(v_{2}\right)\right]+\left[H\left(w_{1}\right)-H\left(w_{2}\right)\right]+\left[H\left(v_{1}\right)-H\left(w_{1}\right)\right]=0,
$$

as required.

We turn now to the statement (15). We give the details for $u \in \Omega_{\delta}^{\bullet}$, the case $w \in \Omega_{\delta}^{\circ}$ being similar. Since $\dot{H}(u)=\frac{2}{\delta} F^{\uparrow}(u) F^{\downarrow}(u)$, we have

$$
\ddot{H}(u)=\frac{2}{\delta}\left(\dot{F}^{\uparrow}(u) F^{\downarrow}(u)+F^{\uparrow}(u) \dot{F}^{\downarrow}(u)\right) .
$$

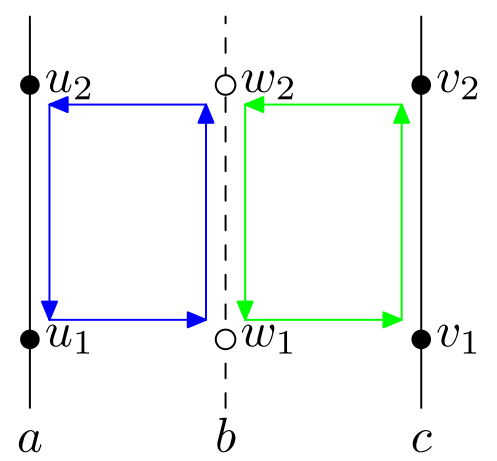

FIG. 5. Contours in the proof of Proposition 3.2. 
Using s-holomorphicity, we deduce that

$$
\begin{gathered}
\ddot{H}(u)=2 \frac{i}{\delta^{2}}\left(\left[F^{\downarrow}(u+\delta / 2)-F^{\downarrow}(u-\delta / 2)\right] F^{\downarrow}(u)\right. \\
\left.+\left[F^{\uparrow}(u+\delta / 2)-F^{\uparrow}(u-\delta / 2)\right] F^{\uparrow}(u)\right) \\
=\frac{i}{\delta^{2}}\left(2 F^{\downarrow}(u-\delta / 2) F^{\downarrow}(u+\delta / 2)-2 F^{\downarrow}(u-\delta / 2)^{2}\right. \\
\left.+2 F^{\uparrow}(u+\delta / 2)^{2}-2 F^{\uparrow}(u-\delta / 2) F^{\uparrow}(u+\delta / 2)\right) .
\end{gathered}
$$

Next,

It follows that

$$
\begin{aligned}
H(u-\delta)-H(u) & =\left|F^{\uparrow}(u-\delta / 2)\right|^{2}-\left|F^{\downarrow}(u-\delta / 2)\right|^{2} \\
& =i\left(F^{\uparrow}(u-\delta / 2)^{2}+F^{\downarrow}(u-\delta / 2)^{2}\right) \text { and } \\
H(u+\delta)-H(u) & =\left|F^{\downarrow}(u+\delta / 2)\right|^{2}-\left|F^{\uparrow}(u+\delta / 2)\right|^{2} \\
& =-i\left(F^{\downarrow}(u+\delta / 2)^{2}+F^{\uparrow}(u+\delta / 2)^{2}\right) .
\end{aligned}
$$

$$
\begin{aligned}
\delta^{2} & \ddot{H}(u)+[H(u-\delta)-H(u)]+[H(u+\delta)-H(u)] \\
\quad & i\left(\left[F^{\uparrow}(u-\delta / 2)-F^{\uparrow}(u+\delta / 2)\right]^{2}-\left[F^{\downarrow}(u-\delta / 2)-F^{\downarrow}(u+\delta / 2)\right]^{2}\right) .
\end{aligned}
$$

Writing $F^{\uparrow}(u-\delta / 2)=a e^{-i \pi / 4}, F^{\uparrow}(u+\delta / 2)=b e^{-i \pi / 4}, F^{\downarrow}(u-\delta / 2)=c e^{i \pi / 4}$, and $F^{\downarrow}(u+\delta / 2)=d e^{i \pi / 4}$, for $a, b, c, d \in \mathbb{R}$, the right-hand-side equals

$$
i\left(\frac{1}{i}[a-b]^{2}-i[c-d]^{2}\right)=(a-b)^{2}+(c-d)^{2} .
$$

But we also have that

$$
\begin{aligned}
& |F(u-\delta / 2)-F(u+\delta / 2)|^{2} \\
& \quad=\left|\left(F^{\uparrow}(u-\delta / 2)-F^{\uparrow}(u+\delta / 2)\right)+\left(F^{\uparrow}(u-\delta / 2)-F^{\uparrow}(u+\delta / 2)\right)\right|^{2} \\
& \quad=(a-b)^{2}+(c-d)^{2}, \text { since } \ell(\uparrow) \perp \ell(\downarrow) .
\end{aligned}
$$

Thus, using also (8),

$$
\delta^{2}\left[\Delta_{\delta} H\right](u)=|F(u-\delta / 2)-F(u+\delta / 2)|^{2}=\delta^{2}|\dot{F}(u)|^{2},
$$

as claimed.

\section{THE FK-OBSERVABLE}

\section{A. Definition}

Let $\left(\Omega_{\delta}, a_{\delta}, b_{\delta}\right)$ be a Dobrushin domain (see Section III A for notation). We will consider FKconfigurations $\xi$ in $\Omega_{\delta}$ and their duals $\xi^{\prime}$. These are defined as in Section II A with some adaptations of the boundary condition. We take $\xi=\xi^{\bullet} \cup \xi^{\circ}$ with $\xi^{\bullet} \subseteq \Omega_{\delta}^{\bullet \text {,int }}$ and $\xi^{\circ} \subseteq \Omega_{\delta}^{\circ \text {,int }}$ finite subsets. Note that we do not allow $\xi^{\bullet}$ to have any points on the black part $\partial_{\delta}^{\bullet}$ of the boundary nor do we allow $\xi^{\circ}$ to have any points on the white part $\partial_{\delta}^{\circ}$. Instead of applying periodic boundary conditions, we let horizontal segments in $\partial_{\delta}^{\bullet}$ and $\partial_{\delta}^{\circ}$ count as primal and dual bridges, respectively. Thus, in essence, we have separately wired together the black and white parts $\partial_{\delta}^{\bullet}$ and $\partial_{\delta}^{\circ}$ of the boundary. See Figure 6 .

An FK-configuration $\xi$ together with its dual $\xi^{\prime}$ define an interface $\gamma$ from $a_{\delta}$ to $b_{\delta}$, separating the (primal) component of $\partial_{\delta}^{\bullet}$ from the (dual) component of $\partial_{\delta}^{\circ}$, and $\gamma$ always has black on the left and white on the right as it travels from $a_{\delta}$ to $b_{\delta}$. We take $\gamma$ to travel in the directions $\uparrow, \downarrow$ on the medial lattice $\mathbb{C}_{\delta}^{\diamond}$ between bridges, and in the directions $\leftarrow, \rightarrow$ at bridges (if $\gamma$ passes the same bridge twice, we slightly separate the points where it passes). We shift $b_{\delta}$ left or right by $\delta / 4$ so that the interface $\gamma$ ends pointing in the direction $\rightarrow$ into $b_{\delta}$. See Figure 6 again.

Apart from the interface $\gamma$, we also draw a loop around each (primal and dual) component which is disjoint from the boundary. We let $L(\xi)$ denote the number of such loops.

Let $\mathbb{E}_{\delta}(\cdot)$ denote the probability measure under which $\xi^{\bullet}$ and $\xi^{\circ}$ are independent Poisson processes on $\Omega_{\delta}^{\bullet \text {,int }}$ and $\Omega_{\delta}^{\circ \text {,int }}$, respectively, both with the same rate $\frac{1}{\delta \sqrt{2}}$. By (2), the density of a random FK-configuration $\xi$ with respect to $\mathbb{E}_{\delta}(\cdot)$ is proportional to

$$
2^{k^{\bullet}(\xi)} h^{\left|\xi^{\bullet}\right|}(2 J)^{\left|\xi^{\circ}\right|}(\delta \sqrt{2})^{\left|\xi^{\bullet}\right|+\left|\xi^{\circ}\right|}
$$



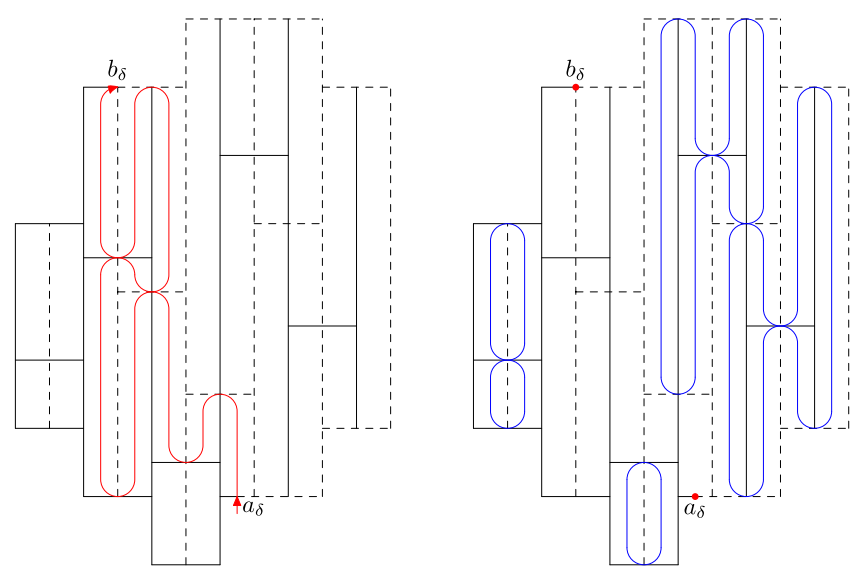

FIG. 6. Dobrushin domain $\left(\Omega_{\delta}, a_{\delta}, b_{\delta}\right)$ with an FK-configuration $\xi$ and its dual $\xi^{\prime}$, as well as the interface $\gamma$ (left) and the $L(\xi)=5$ loops (right). We have omitted the $\times$-marks for cuts.

Using the Euler-relation, one may see that $k^{\bullet}(\xi)-\left|\xi^{\bullet}\right|=k^{\circ}(\xi)-\left|\xi^{\circ}\right|+$ cst for some constant not depending on $\xi$. Also, $L(\xi)=k^{\bullet}(\xi)+k^{\circ}(\xi)-2$. We choose the parameters

$$
h=J=\frac{1}{2 \delta} \text {. }
$$

It then follows that the density (16) is proportional to simply $(\sqrt{2})^{L(\xi)}$. We write $\hat{\mathbb{E}}_{\delta}=\hat{\mathbb{E}}_{\left(\Omega_{\delta}, a_{\delta}, b_{\delta}\right)}$ for the critical FK-law in $\left(\Omega_{\delta}, a_{\delta}, b_{\delta}\right)$ given by

$$
\frac{d \hat{\mathbb{E}}_{\delta}}{d \mathbb{E}_{\delta}}(\xi)=\frac{(\sqrt{2})^{L(\xi)}}{Z_{\delta}}, \text { where } Z_{\delta}=\mathbb{E}_{\delta}\left[(\sqrt{2})^{L(\xi)}\right] .
$$

Now let $z \in \Omega_{\delta}^{\bullet} \cup \Omega_{\delta}^{\circ}$ be arbitrary. For $\alpha \in\{\uparrow, \downarrow, \leftarrow, \rightarrow\}$, define the event

$$
\Gamma_{z}^{\alpha}=\{\xi: \gamma(\xi) \text { passes by } z \text { in direction } \alpha\} .
$$

For $\alpha \in\{\uparrow, \downarrow\}$, we count both the case when $\gamma$ passes on the left side of $z$ (i.e., goes through $z-\delta / 4$ ) and when it passes on the right side (i.e., goes through $z+\delta / 4$ ). Similarly, for $\alpha \in\{\leftarrow, \rightarrow\}$, we count both the cases when $\gamma$ passes "just below" $z$ and "just above" $z$.

Assuming that $\Gamma_{z}^{\alpha}$ happens, let $W_{\gamma}^{\alpha}(z)$ denote the winding-angle (in radians) of $\gamma$ from $z$ to the exit $b_{\delta}$; if $\gamma$ passes $z$ twice, in opposite directions, we count here the winding angle from when it passes in direction $\alpha$. Note that $W_{\gamma}^{\alpha}(z)$ is deterministic up to a multiple of $2 \pi$.

We define the four (random) functions $\varphi^{\uparrow}(\xi ; z), \varphi^{\downarrow}(\xi ; z), \varphi^{\leftarrow}(\xi ; z)$, and $\varphi^{\rightarrow}(\xi ; z)$ by

$$
\varphi^{\alpha}(\xi ; z)=\mathbb{I}_{\Gamma_{z}^{\alpha}}(\xi) \exp \left(\frac{i}{2} W_{\gamma(\xi)}^{\alpha}(z)\right) .
$$

Note that the supports of $\varphi^{\leftarrow}(\xi ; z)$ and of $\varphi^{\rightarrow}(\xi ; z)$ are discrete sets contained in $\xi \cup \partial^{\mathrm{h}} \Omega_{\delta}$, whereas the supports of $\varphi^{\uparrow}(\xi ; z)$ and $\varphi^{\downarrow}(\xi ; z)$ are disjoint from $\xi$. Also note that if $u \in \Omega_{\delta}^{\bullet}$ is black and $w=u+\delta / 2$ is the white neighbour of $u$ on the right, then $\varphi^{\uparrow}(\xi ; u)=\varphi^{\uparrow}(\xi ; w)$, whereas if $w^{\prime}=u-\delta / 2$ is the white neighbour of $u$ on the left, then $\varphi^{\downarrow}(\xi ; u)=\varphi^{\downarrow}\left(\xi ; w^{\prime}\right)$. (Here we assume that $u \pm \delta / 2 \in \Omega_{\delta}^{\circ}$ in the appropriate cases.)

\section{Definition 4.1. Write}

$$
\Phi_{\delta}^{\uparrow}(z)=\hat{\mathbb{E}}_{\delta}\left[\varphi^{\uparrow}(\xi ; z)\right], \quad \Phi_{\delta}^{\downarrow}(z)=\hat{\mathbb{E}}_{\delta}\left[\varphi^{\downarrow}(\xi ; z)\right] .
$$

We define the $\mathrm{FK}-$ Ising observable $F_{\delta}(z)=F_{\delta}^{\mathrm{FK}}(z)$ by

$$
F_{\delta}(z)=\Phi_{\delta}^{\uparrow}(z)+\Phi_{\delta}^{\downarrow}(z), \quad z \in \Omega_{\delta}^{\bullet} \cup \Omega_{\delta}^{\circ} .
$$

We remark that the notation used here is consistent with our previous notation (7) for the projections $F^{\uparrow}, F^{\downarrow}$ of a function $F$ onto $\ell(\uparrow)=e^{-i \pi / 4} \mathbb{R}$ and $\ell(\downarrow)=e^{i \pi / 4} \mathbb{R}$, in the sense that

$$
F_{\delta}^{\uparrow}(z)=\Phi_{\delta}^{\uparrow}(z) \text { and } F_{\delta}^{\downarrow}(z)=\Phi_{\delta}^{\downarrow}(z) .
$$


Indeed, if we identify arrows $\alpha \in\{\uparrow, \downarrow, \leftarrow, \rightarrow\}$ with complex numbers by the rules

$$
\rightarrow=1=i^{0}, \quad \uparrow=i=i^{1}, \quad \leftarrow=-1=i^{2}, \quad \downarrow=-i=i^{3},
$$

then we have that

$$
W_{\gamma(\xi)}^{\alpha}(z)=-\arg (\alpha)+2 \pi n(\xi)
$$

for some random $n(\xi) \in \mathbb{Z}$. Thus $\varphi^{\alpha}(\xi ; z)$ is a real multiple of $\sqrt{\bar{\alpha}}$, i.e., it belongs to $\ell(\alpha)$. Note that the line $\sqrt{\bar{\alpha}} \mathbb{R}$ does not depend on the choice of square root.

\section{B. Comparison with isoradial graphs}

For readers familiar with the work of Chelkak and Smirnov ${ }^{12}$ on the classical Ising model on isoradial graphs, the following brief discussion may be useful. Let $0<\varepsilon \ll \delta$ and consider a rhombic tiling of $\mathbb{C}$ where all the rhombi have two vertices in each of $\mathbb{C}_{\delta}^{\bullet}$ and $\mathbb{C}_{\delta}^{\circ}$, and acute angle $2 \varepsilon$, as in Figure 7. This corresponds to an isoradial embedding of $\mathbb{Z}^{2}$ with common radius $\frac{\delta}{2 \cos (\varepsilon)}$ and vertices restricted to $\mathbb{C}_{\delta}^{\bullet}$.

Let $\hat{\mathbb{E}}_{\delta, \varepsilon}(\cdot)$ denote the law of the critical (classical) FK-Ising model in some Dobrushin-domain in this graph, as given, for example, in Eq. (2.1) of Ref. 12. Thus each rhombus either contains a vertical or a horizontal edge between two of its vertices. It is well-known that the laws $\hat{\mathbb{E}}_{\delta, \varepsilon}$ converge weakly to $\hat{\mathbb{E}}_{\delta}$ as $\varepsilon \rightarrow 0$. (To make this convergence precise, one can identify a configuration with the collection of rhombus centres $z$ for which the rhombus contains a horizontal edge, and view this as a point process in $\mathbb{C}_{\delta}^{\bullet} \cup \mathbb{C}_{\delta}^{\circ}$. The weak convergence is then in the standard sense for simple point processes, see, e.g., Ref. 14, Chapter 11.1.)

In this setting, the interface $\gamma_{\varepsilon}$ is taken to cross the rhombus-sides perpendicularly, i.e., roughly speaking in the directions $\nearrow, \searrow, \nwarrow$, and $\swarrow$. If we specify a rhombus as well as one of these four directions of travel, this corresponds to a unique edge of the rhombus, hence the edge-observables [Ref. 12, Eq. (2.2)] of Chelkak and Smirnov can be indexed as $F_{\delta, \varepsilon}^{\nearrow}(z), F_{\delta, \varepsilon}^{\searrow}(z), \ldots$ for rhombus centres $z$. Using notation similar to (18), we have (up to a real factor)

$$
F_{\delta, \varepsilon}^{\alpha}(z)=\hat{\mathbb{E}}_{\delta, \varepsilon}\left[\mathbb{I}_{\Gamma_{z}^{\alpha}} \exp \left(\frac{i}{2} W_{\gamma_{\varepsilon}}^{\alpha}(z)\right)\right], \quad \alpha \in\{\nearrow, \searrow, \nwarrow, \swarrow\}
$$

We may further take $\gamma_{\varepsilon}$ to pass "closest" to rhombus centres $z$ in the directions $\uparrow, \downarrow$, $\leftarrow$, or $\rightarrow$. This allows us to define more observables,

$$
\Phi_{\delta, \varepsilon}^{\alpha}(z)=\hat{\mathbb{E}}_{\delta, \varepsilon}\left[\mathbb{I}_{\Gamma_{z}^{\alpha}} \exp \left(\frac{i}{2} W_{\gamma_{\varepsilon}}^{\alpha}(z)\right)\right], \quad \alpha \in\{\uparrow, \downarrow, \leftarrow, \rightarrow\}
$$

Clearly each $\Phi_{\delta, \varepsilon}^{\alpha}(z) \in \ell(\alpha)$ as is the case for the $\Phi_{\delta}^{\alpha}(z)$ (provided we assume that $\gamma_{\varepsilon}$ exits the domain in the direction $\rightarrow$ ).

Referring to Figure 7, we see, for example, that if $\gamma_{\varepsilon}$ enters the rhombus of $z$ in direction $\nwarrow$ (edge on the lower right of $z$ ), then it passes closest to $z$ in either direction $\uparrow$ as depicted, or direction $\leftarrow$, but not both ( $\uparrow$ if there is a black vertical edge at $z$, and $\leftarrow$ if there is a white horizontal edge). Similar considerations apply at all rhombus centres, and this allows us to derive linear relations for

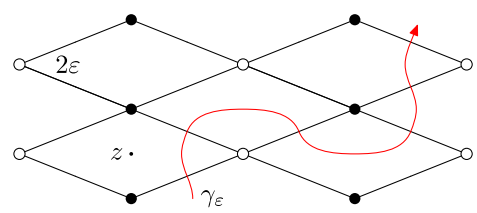

FIG. 7. Isoradial approximation of the FK-representation of the TFIM. 
the $F_{\delta, \varepsilon}^{\alpha}(z)$ in terms of the $\Phi_{\delta, \varepsilon}^{\alpha}(z)$. Writing $\varepsilon^{\star}=\frac{\pi}{2}-\varepsilon$, we have

$$
\left(\begin{array}{c}
F_{\delta, \varepsilon}^{\nwarrow}(z) \\
F_{\delta, \varepsilon}^{\nearrow}(z) \\
F_{\delta, \varepsilon}^{\searrow}(z) \\
F_{\delta, \varepsilon}^{\swarrow}(z)
\end{array}\right)=\left(\begin{array}{cccc}
e^{-\frac{i}{2} \varepsilon} & 0 & 0 & e^{\frac{i}{2} \varepsilon^{\star}} \\
e^{\frac{i}{2} \varepsilon} & e^{-\frac{i}{2} \varepsilon^{\star}} & 0 & 0 \\
0 & e^{\frac{i}{2} \varepsilon^{\star}} & e^{-\frac{i}{2} \varepsilon} & 0 \\
0 & 0 & e^{\frac{i}{2} \varepsilon} & e^{-\frac{i}{2} \varepsilon^{\star}}
\end{array}\right)\left(\begin{array}{c}
\Phi_{\delta, \varepsilon}^{\uparrow}(z) \\
\Phi_{\delta, \varepsilon}^{\overrightarrow{1}}(z) \\
\Phi_{\delta, \varepsilon}^{\downarrow}(z) \\
\Phi_{\delta, \varepsilon}^{\leftarrow}(z)
\end{array}\right) .
$$

The FK-Ising observable [Ref. 12, Eq. (2.4)] of Chelkak and Smirnov is given by

$$
\begin{aligned}
F_{\delta, \varepsilon}(z) & =\frac{1}{2} \sum_{\alpha} F_{\delta, \varepsilon}^{\alpha}(z) \\
& =\cos (\varepsilon / 2)\left[\Phi_{\varepsilon}^{\uparrow}(z)+\Phi_{\varepsilon}^{\downarrow}(z)\right]+\cos \left(\varepsilon^{\star} / 2\right)\left[\Phi_{\varepsilon}^{\leftarrow}(z)+\Phi_{\varepsilon}^{\rightarrow}(z)\right],
\end{aligned}
$$

where the second line uses (21). Assuming the existence of the limits

$$
\Phi_{\delta}^{\uparrow}(z)=\lim _{\varepsilon \rightarrow 0} \Phi_{\delta, \varepsilon}^{\uparrow}(z), \quad \Phi_{\delta}^{\downarrow}(z)=\lim _{\varepsilon \rightarrow 0} \Phi_{\delta, \varepsilon}^{\downarrow}(z),
$$

as well as $\Phi_{\delta, \varepsilon}^{\alpha}(z)=O(\varepsilon)$ for $\alpha \in\{\leftarrow, \rightarrow\}$, we get

$$
\lim _{\varepsilon \rightarrow 0} F_{\delta, \varepsilon}(z)=\Phi_{\delta}^{\uparrow}(z)+\Phi_{\delta}^{\downarrow}(z),
$$

which is how we defined our observable $F_{\delta}(z)$.

\section{S-holomorphicity}

In this section, we show the following result.

Theorem 4.2. Let $F_{\delta}=F_{\delta}^{\mathrm{FK}}$ be the $\mathrm{FK}-$ observable in a Dobrushin domain $\left(\Omega_{\delta}, a_{\delta}, b_{\delta}\right)$. Then $F_{\delta}$ is s-holomorphic in $\Omega_{\delta}$.

It is immediate that $F_{\delta}$ satisfies conditions (9) and (10) in the definition of s-holomorphicity, see the discussion just above Definition 4.1. We thus need to show that also (11) is satisfied. In the proof, we drop the subscript $\delta$ from $\mathbb{E}$ and $\hat{\mathbb{E}}$.

For $z \in \Omega_{\delta}^{\bullet, \text { int }} \cup \Omega_{\delta}^{\circ \text {,int }}$, we let $\xi_{z}=\xi \triangle\{z\}$ and we define the auxiliary observables

$$
\begin{aligned}
& \Phi_{\delta}^{\leftarrow}(z)=\hat{\mathbb{E}}\left[(\sqrt{2})^{L\left(\xi_{z}\right)-L(\xi)} \varphi^{\leftarrow}\left(\xi_{z} ; z\right)\right] \\
& \Phi_{\delta}^{\vec{S}}(z)=\hat{\mathbb{E}}\left[(\sqrt{2})^{L\left(\xi_{z}\right)-L(\xi)} \varphi^{\rightarrow}\left(\xi_{z} ; z\right)\right]
\end{aligned}
$$

If $z \in \partial^{\mathrm{v}} \Omega_{\delta}$ is in the vertical part of the boundary, then we set $\Phi_{\delta}^{\leftarrow}(z)=\Phi_{\delta}^{\overrightarrow{ }}(z)=0$ (we will not need to define them for $\left.z \in \partial^{\mathrm{h}} \Omega_{\delta}\right)$. As we remarked above, we have that $\Phi_{\delta}(z) \in \ell(\rightarrow)=\mathbb{R}$ and that $\Phi_{\delta}^{\leftarrow}(z) \in \ell(\leftarrow)=i \mathbb{R}$. We now claim the following.

Lemma 4.3. For all $z \in \Omega_{\delta}^{\bullet \text {,int }} \cup \Omega_{\delta}^{\circ \text {,int }}$, we have that

$$
\begin{aligned}
& \Phi_{\delta}^{\uparrow}(z)=\frac{1}{\sqrt{2}}\left(e^{i \pi / 4} \Phi_{\delta}^{\leftarrow}(z)+e^{-i \pi / 4} \Phi_{\delta}^{\rightarrow}(z)\right), \\
& \Phi_{\delta}^{\downarrow}(z)=\frac{1}{\sqrt{2}}\left(e^{i \pi / 4} \Phi_{\delta}^{\rightarrow}(z)+e^{-i \pi / 4} \Phi_{\delta}^{\leftarrow}(z)\right) .
\end{aligned}
$$

Proof. We prove the statement for $\Phi_{\delta}^{\uparrow}(z)$ in the case when $z \in \Omega_{\delta}^{\circ \text {,int }}$ is white, the other case $z \in \Omega_{\delta}^{\bullet, \text { int }}$ is similar. We refer to Figures $8-10$.

Let $A$ denote the event that $\gamma$ passes $z$ only once, in the direction $\uparrow$, as depicted on the left in Figure 8. Let $A^{\prime}$ denote the event that $\gamma$ passes $z$ once in the direction $\rightarrow$ and once in the direction $\leftarrow$, with $\rightarrow$ coming first, as depicted on the right in Figure 8. Similarly, let $B$ and $B^{\prime}$ denote the events depicted in Figure 9. Explicitly, $B$ is the event that $\gamma$ passes $z$ both going $\uparrow$ and $\downarrow$, with $\downarrow$ coming first, and $B^{\prime}$ is the event that $\gamma$ passes $z$ in direction $\leftarrow$ only. Finally, let $C$ and $C^{\prime}$ be as in Figure 10: $C$ is the event that $\gamma$ passes $z$ in direction $\uparrow$ and later in direction $\downarrow$, and $C^{\prime}$ is the event that it passes in direction $\rightarrow$ only. 

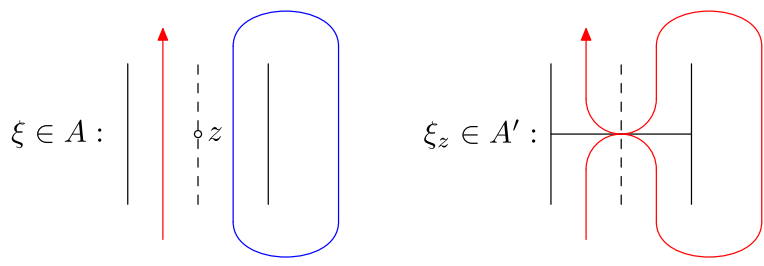

FIG. 8. In $\xi$, the interface $\gamma$ passes $z$ in direction $\uparrow$ only and in $\xi_{z}$, it passes in directions $\rightarrow$ and $\leftarrow$.
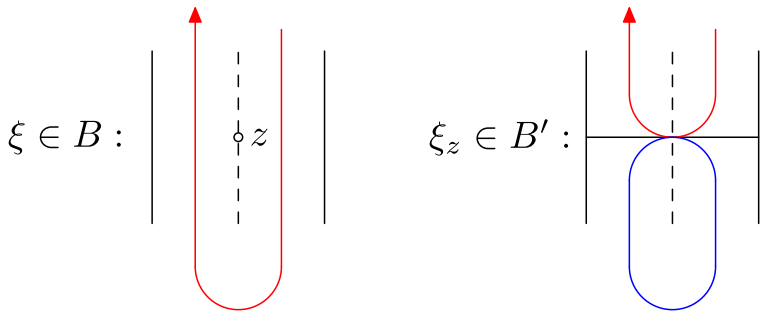

FIG. 9. In $\xi$, the interface $\gamma$ passes $z$ in directions $\uparrow$ and $\downarrow$ and in $\xi_{z}$, it passes in direction $\leftarrow$.

We note the following facts. First,

$$
\begin{aligned}
& \xi \in A \Leftrightarrow \xi_{z} \in A^{\prime}, \text { and then } L(\xi)=L\left(\xi_{z}\right)+1, \\
& \xi \in B \Leftrightarrow \xi_{z} \in B^{\prime}, \text { and then } L(\xi)=L\left(\xi_{z}\right)-1, \\
& \xi \in C \Leftrightarrow \xi_{z} \in C^{\prime}, \text { and then } L(\xi)=L\left(\xi_{z}\right)-1 .
\end{aligned}
$$

Second, the event $\Gamma_{z}^{\uparrow}=\{\gamma$ passes $z$ going $\uparrow\}$ satisfies

$$
\mathbb{I}_{\Gamma_{z}^{\uparrow}}(\xi)=\mathbb{I}_{A}(\xi)+\mathbb{1}_{B}(\xi)+\mathbb{I}_{C}(\xi)
$$

and the events $\Gamma_{z}^{\leftarrow}$ and $\Gamma_{z}^{\rightarrow}$ satisfy

$$
\begin{aligned}
& \mathbb{I}_{\Gamma_{z}^{\leftarrow}}\left(\xi_{z}\right)=\mathbb{I}_{A^{\prime}}\left(\xi_{z}\right)+\mathbb{I}_{B^{\prime}}\left(\xi_{z}\right) \text { and } \\
& \mathbb{I}_{\Gamma_{z}^{\rightarrow}}\left(\xi_{z}\right)=\mathbb{I}_{A^{\prime}}\left(\xi_{z}\right)+\mathbb{I}_{C^{\prime}}\left(\xi_{z}\right) .
\end{aligned}
$$

Third, the winding angles are related by

$$
\begin{aligned}
& W_{\gamma(\xi)}^{\uparrow}(z)=W_{\gamma\left(\xi_{z}\right)}^{\leftarrow}(z)+\pi / 2, \text { for } \xi \in A \cup B, \\
& W_{\gamma(\xi)}^{\uparrow}(z)=W_{\gamma\left(\xi_{z}\right)}^{\rightarrow}(z)-\pi / 2, \text { for } \xi \in A \cup C .
\end{aligned}
$$

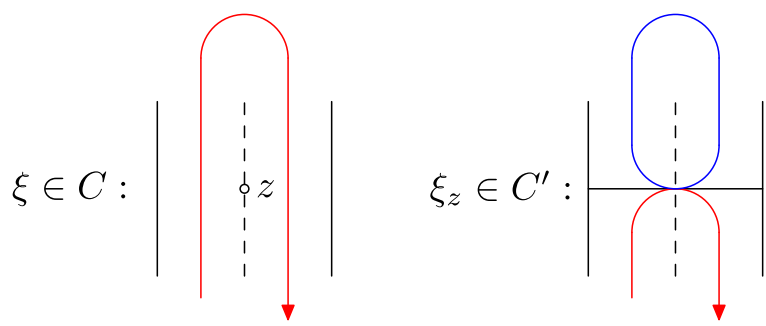

FIG. 10. In $\xi$, the interface $\gamma$ passes $z$ in directions $\uparrow$ and $\downarrow$ and in $\xi_{z}$, it passes in direction $\rightarrow$ 
Using these facts, we obtain

$$
\begin{aligned}
\varphi^{\uparrow}(\xi ; z)= & \left(\mathbb{I}_{A}(\xi)+\mathbb{I}_{B}(\xi)+\mathbb{I}_{C}(\xi)\right) \exp \left(\frac{i}{2} W_{\gamma(\xi)}^{\uparrow}(z)\right) \\
= & \frac{1}{2} \mathbb{I}_{A^{\prime}}\left(\xi_{z}\right) \exp \left(\frac{i}{2} W_{\gamma\left(\xi_{z}\right)}^{\leftarrow}(z)\right) e^{i \pi / 4} \\
& +\frac{1}{2} \mathbb{I}_{A^{\prime}}\left(\xi_{z}\right) \exp \left(\frac{i}{2} W_{\gamma\left(\xi_{z}\right)}(z)\right) e^{-i \pi / 4} \\
& +\mathbb{I}_{B^{\prime}}\left(\xi_{z}\right) \exp \left(\frac{i}{2} W_{\gamma\left(\xi_{z}\right)}^{\leftarrow}(z)\right) e^{i \pi / 4} \\
& +\mathbb{I}_{C^{\prime}}\left(\xi_{z}\right) \exp \left(\frac{i}{2} W_{\gamma\left(\xi_{z}\right)}^{\rightarrow}(z)\right) e^{-i \pi / 4}
\end{aligned}
$$

Thus

$$
\begin{aligned}
(\sqrt{2})^{L(\xi)} \varphi^{\uparrow}(\xi ; z) \\
=(\sqrt{2})^{L(\xi)-2} \mathbb{I}_{A^{\prime}}\left(\xi_{z}\right)\left\{\exp \left(\frac{i}{2} W_{\gamma\left(\xi_{z}\right)}^{\leftarrow}(z)\right) e^{i \pi / 4}+\exp \left(\frac{i}{2} W_{\gamma\left(\xi_{z}\right)}(z)\right) e^{-i \pi / 4}\right\} \\
\quad+(\sqrt{2})^{L(\xi)} \mathbb{I}_{B^{\prime}}\left(\xi_{z}\right) \exp \left(\frac{i}{2} W_{\gamma\left(\xi_{z}\right)}^{\leftarrow}(z)\right) e^{i \pi / 4} \\
\quad+(\sqrt{2})^{L(\xi)} \mathbb{I}_{C^{\prime}}\left(\xi_{z}\right) \exp \left(\frac{i}{2} W_{\gamma\left(\xi_{z}\right)}(z)\right) e^{-i \pi / 4} \\
=(\sqrt{2})^{L\left(\xi_{z}\right)-1}\left[\varphi^{\leftarrow}\left(\xi_{z} ; z\right) e^{i \pi / 4}+\varphi^{\rightarrow}\left(\xi_{z} ; z\right) e^{-i \pi / 4}\right] .
\end{aligned}
$$

Taking the $\mathbb{E}$-expectation,

$$
\begin{aligned}
& \mathbb{E}\left[(\sqrt{2})^{L(\xi)} \varphi^{\uparrow}(\xi ; z)\right] \\
& \quad=\frac{1}{\sqrt{2}}\left(\mathbb{E}\left[(\sqrt{2})^{L\left(\xi_{z}\right)} \varphi^{\leftarrow}\left(\xi_{z} ; z\right)\right] e^{i \pi / 4}+\mathbb{E}\left[(\sqrt{2})^{L\left(\xi_{z}\right)} \varphi^{\rightarrow}\left(\xi_{z} ; z\right)\right] e^{-i \pi / 4}\right) .
\end{aligned}
$$

This readily gives the claim (23) for $\Phi^{\uparrow}(z)$.

We now calculate $\dot{\Phi}_{\delta}^{\uparrow}$ and $\dot{\Phi}_{\delta}^{\downarrow}$. We will use the notation $\xi(z, z+i \varepsilon)$ for the number of elements of $\xi$ in the interval $(z, z+i \varepsilon)$. For a function $f(\xi, z)$, we write $f(\xi, t \pm)=\lim _{\varepsilon \downarrow 0} f(\xi, t \pm i \varepsilon)$. Recall that $\xi_{z}=\xi \Delta\{z\}$.

Lemma 4.4. Let $w \in \Omega_{\delta}^{\circ, \text { int }}$ and write $u=w-\delta / 2$ and $v=w+\delta / 2$. Then

$$
\begin{aligned}
\dot{\Phi}_{\delta}^{\uparrow}(w)=\dot{\Phi}_{\delta}^{\uparrow}(u)= & \frac{1}{\delta \sqrt{2}}\left(e^{i \pi / 4} \Phi_{\delta}^{\leftarrow}(w)-e^{-i \pi / 4} \Phi_{\delta}^{\overrightarrow{ }}(w)\right) \\
& +\frac{1}{\delta \sqrt{2}}\left(e^{-i \pi / 4} \Phi_{\delta}^{\overrightarrow{ }}(u)-e^{i \pi / 4} \Phi_{\delta}^{\leftarrow}(u)\right)
\end{aligned}
$$

and

$$
\begin{aligned}
\dot{\Phi}_{\delta}^{\downarrow}(w)=\dot{\Phi}_{\delta}^{\downarrow}(v)= & \frac{1}{\delta \sqrt{2}}\left(e^{-i \pi / 4} \Phi_{\delta}^{\leftarrow}(w)-e^{i \pi / 4} \Phi_{\delta}^{\overrightarrow{ }}(w)\right) \\
& +\frac{1}{\delta \sqrt{2}}\left(e^{i \pi / 4} \Phi_{\delta}(v)-e^{-i \pi / 4} \Phi_{\delta}^{\leftarrow}(v)\right)
\end{aligned}
$$

Proof. The first equalities in (25) and (26) hold since $\Phi_{\delta}^{\uparrow}(w)=\Phi_{\delta}^{\uparrow}(u)$ and $\Phi_{\delta}^{\downarrow}(w)=\Phi_{\delta}^{\downarrow}(v)$. We prove (25) and the other claim (26) is similar. Let $\varepsilon>0$ (the case $\varepsilon<0$ is similar). We have that

$$
Z_{\delta} \frac{\Phi_{\delta}^{\uparrow}(w+i \varepsilon)-\Phi_{\delta}^{\uparrow}(w)}{\varepsilon}=\frac{1}{\varepsilon} \mathbb{E}\left[(\sqrt{2})^{L(\xi)}\left(\varphi^{\uparrow}(\xi ; w+i \varepsilon)-\varphi^{\uparrow}(\xi ; w)\right)\right] .
$$

Note that $\varphi^{\uparrow}(\xi ; w+i \varepsilon)-\varphi^{\uparrow}(\xi ; w)=0$ unless either $\xi(w, w+i \varepsilon)>0$ or $\xi(u, u+i \varepsilon)>0$. The probability that both these happen is $O\left(\varepsilon^{2}\right)$ and may therefore be ignored. Also recall that $\varphi^{\uparrow}(\xi ; w)=\varphi^{\uparrow}(\xi ; u)$ for $w$ and $u$ as specified. Thus the right-hand-side of (27) equals

$$
\begin{aligned}
& \frac{1}{\varepsilon} \mathbb{E}\left[(\sqrt{2})^{L(\xi)}\left(\varphi^{\uparrow}(\xi ; w+i \varepsilon)-\varphi^{\uparrow}(\xi ; w)\right) \mathbb{I}\{\xi(w, w+i \varepsilon)>0\}\right] \\
& \quad+\frac{1}{\varepsilon} \mathbb{E}\left[(\sqrt{2})^{L(\xi)}\left(\varphi^{\uparrow}(\xi ; u+i \varepsilon)-\varphi^{\uparrow}(\xi ; u)\right) \mathbb{I}\{\xi(u, u+i \varepsilon)>0\}\right]+o(1) .
\end{aligned}
$$

As $\varepsilon \rightarrow 0$, this converges to

$$
\begin{aligned}
& \frac{1}{\delta \sqrt{2}} \mathbb{E}\left[(\sqrt{2})^{L\left(\xi_{w}\right)}\left(\varphi^{\uparrow}\left(\xi_{w} ; w+\right)-\varphi^{\uparrow}\left(\xi_{w} ; w-\right)\right)\right] \\
& +\frac{1}{\delta \sqrt{2}} \mathbb{E}\left[(\sqrt{2})^{L\left(\xi_{u}\right)}\left(\varphi^{\uparrow}\left(\xi_{u} ; u+\right)-\varphi^{\uparrow}\left(\xi_{u} ; u-\right)\right)\right] .
\end{aligned}
$$


Here we used that the conditional distribution of $\xi$ given $\xi(w, w+i \varepsilon)>0$ converges to the law of $\xi_{w}$.

Consider $\varphi^{\uparrow}\left(\xi_{w} ; w+\right)-\varphi^{\uparrow}\left(\xi_{w} ; w-\right)$. We refer again to Figures 8-10 and the events $A, B, C, A^{\prime}, B^{\prime}, C^{\prime}$ depicted there, as well as the relation (24) between winding angles. We have that

$$
\begin{array}{r}
\text { for } \xi \in A, \quad \varphi^{\uparrow}\left(\xi_{w} ; w+\right)-\varphi^{\uparrow}\left(\xi_{w} ; w-\right)=0 \\
=\varphi^{\leftarrow}\left(\xi_{w} ; w\right) e^{i \pi / 4}-\varphi^{\rightarrow}\left(\xi_{w} ; w\right) e^{-i \pi / 4}, \\
\text { for } \xi \in B, \quad \varphi^{\uparrow}\left(\xi_{w} ; w+\right)-\varphi^{\uparrow}\left(\xi_{w} ; w-\right)=\varphi^{\uparrow}\left(\xi_{w} ; w+\right) \\
=\varphi^{\leftarrow}\left(\xi_{w} ; w\right) e^{i \pi / 4} \\
=\varphi^{\leftarrow}\left(\xi_{w} ; w\right) e^{i \pi / 4}-\varphi^{\rightarrow}\left(\xi_{w} ; w\right) e^{-i \pi / 4}, \\
\text { for } \xi \in C, \quad \varphi^{\uparrow}\left(\xi_{w} ; w+\right)-\varphi^{\uparrow}\left(\xi_{w} ; w-\right)=-\varphi^{\uparrow}\left(\xi_{w} ; w-\right) \\
=-\varphi^{\rightarrow}\left(\xi_{w} ; w\right) e^{-i \pi / 4} \\
=\varphi^{\leftarrow}\left(\xi_{w} ; w\right) e^{i \pi / 4}-\varphi^{\rightarrow}\left(\xi_{w} ; w\right) e^{-i \pi / 4} .
\end{array}
$$

That is to say, we have the identity

$$
\varphi^{\uparrow}\left(\xi_{w} ; w+\right)-\varphi^{\uparrow}\left(\xi_{w} ; w-\right)=\varphi^{\leftarrow}\left(\xi_{w} ; w\right) e^{i \pi / 4}-\varphi^{\rightarrow}\left(\xi_{w} ; w\right) e^{-i \pi / 4} .
$$

This gives

$$
\mathbb{E}\left[(\sqrt{2})^{L\left(\xi_{w}\right)}\left(\varphi^{\uparrow}\left(\xi_{w} ; w+\right)-\varphi^{\uparrow}\left(\xi_{w} ; w-\right)\right)\right]=Z_{\delta}\left(\Phi_{\delta}^{\leftarrow}(w) e^{i \pi / 4}-\Phi_{\delta}(w) e^{-i \pi / 4}\right)
$$

Similar considerations give

$$
\mathbb{E}\left[(\sqrt{2})^{L\left(\xi_{u}\right)}\left(\varphi^{\uparrow}\left(\xi_{u} ; u+\right)-\varphi^{\uparrow}\left(\xi_{u} ; u-\right)\right)\right]=Z_{\delta}\left(\Phi_{\delta}^{\rightarrow}(w) e^{-i \pi / 4}-\Phi_{\delta}^{\leftarrow}(w) e^{i \pi / 4}\right) .
$$

Combining these and dividing by $Z_{\delta}$ give the claim (25).

Proof of Theorem 4.2. As already noted, properties (9) and (10) are immediate, so we need to establish (11). We check the case $z=w \in \Omega_{\delta}^{\circ \text {,int }}$, the case $z \in \Omega_{\delta}^{\bullet, \text { int }}$ being similar. Writing $u=w-\delta / 2$ and $v=w+\delta / 2$, we need to show that

$$
\begin{aligned}
& \dot{\Phi}_{\delta}^{\uparrow}(w)=\dot{\Phi}_{\delta}^{\uparrow}(u)=\frac{i}{\delta}\left(\Phi_{\delta}^{\downarrow}(w)-\Phi_{\delta}^{\downarrow}(u)\right), \\
& \dot{\Phi}_{\delta}^{\downarrow}(w)=\dot{\Phi}_{\delta}^{\downarrow}(v)=\frac{i}{\delta}\left(\Phi_{\delta}^{\uparrow}(v)-\Phi_{\delta}^{\uparrow}(w)\right) .
\end{aligned}
$$

But for any $z \in \Omega_{\delta}^{\circ \text {,int }} \cup \Omega_{\delta}^{\bullet \text {,int }}$, we have, by Lemma 4.3 , first

$$
\begin{aligned}
e^{i \pi / 4} \Phi_{\delta}^{\leftarrow}(z)-e^{-i \pi / 4} \Phi_{\delta}(z) & =e^{i \pi / 2} e^{-i \pi / 4} \Phi_{\delta}^{\leftarrow}(z)-e^{-i \pi / 2} e^{i \pi / 4} \Phi_{\delta}(z) \\
& =i \cdot\left(e^{-i \pi / 4} \Phi_{\delta}^{\leftarrow}(z)+e^{i \pi / 4} \Phi_{\delta}(z)\right) \\
& =i \sqrt{2} \cdot \Phi_{\delta}^{\downarrow}(z),
\end{aligned}
$$

and second

$$
\begin{aligned}
e^{i \pi / 4} \Phi_{\delta}^{\vec{~}}(z)-e^{-i \pi / 4} \Phi_{\delta}^{\leftarrow}(z) & =e^{i \pi / 2} e^{-i \pi / 4} \Phi_{\delta}^{\overrightarrow{ }}(z)-e^{-i \pi / 2} e^{i \pi / 4} \Phi_{\delta}^{\leftarrow}(z) \\
& =i \cdot\left(e^{-i \pi / 4} \Phi_{\delta}^{\overrightarrow{ }}(z)+e^{i \pi / 4} \Phi_{\delta}^{\leftarrow}(z)\right) \\
& =i \sqrt{2} \cdot \Phi_{\delta}^{\uparrow}(z) .
\end{aligned}
$$

Putting these into Lemma 4.4 gives the result.

\section{THE SPIN-OBSERVABLE}

\section{A. Definition}

Let $\Omega_{\delta}$ be a discrete dual domain (see Section III A). We work with the random-parity representation (3) in $\Omega_{\delta}^{\bullet}$, and as before we set $h=J=\frac{1}{2 \delta}$. Recall that the set $\xi=\xi^{\circ} \subseteq \Omega_{\delta}^{\circ}$ of bridges is a Poisson process with rate $J$. Define the "lower boundary" of $\Omega_{\delta}^{\bullet}$ as

$$
\partial^{-} \Omega_{\delta}^{\bullet}=\left\{z \in \partial \Omega_{\delta}^{\bullet}: z-i \varepsilon \notin \Omega_{\delta}^{\bullet} \text { for all } \varepsilon>0 \text { small enough }\right\},
$$


and similarly the "upper boundary" of $\Omega_{\delta}^{\bullet}$ as

$$
\partial^{+} \Omega_{\delta}^{\bullet}=\left\{z \in \partial \Omega_{\delta}^{\bullet}: z+i \varepsilon \notin \Omega_{\delta}^{\bullet} \text { for all } \varepsilon>0 \text { small enough }\right\} .
$$

Thus $\partial^{-} \Omega_{\delta}^{\bullet} \cup \partial^{+} \Omega_{\delta}^{\bullet}=\partial^{\mathrm{h}} \Omega_{\delta}^{\bullet}$.

We take two distinct points $a_{\delta}, b_{\delta}$ on the boundary $\partial \Omega_{\delta}$ with $a_{\delta} \in \partial^{\mathrm{v}} \Omega_{\delta}^{\circ} \cup \partial^{\mathrm{h}} \Omega_{\delta}^{\bullet}$ either a white point on the "sides" or a black point on the "top or bottom," and $b_{\delta} \in \partial^{-} \Omega_{\delta}^{\circ}$ on the lower boundary. In the case when $a_{\delta} \in \partial^{\mathrm{v}} \Omega_{\delta}^{\circ}$, we let $a_{\delta}^{\text {int }}=a_{\delta} \pm \delta / 2 \in \Omega_{\delta}^{\bullet}$ be the black point in $\Omega_{\delta}$ next to $a_{\delta}$, so that $\left(a_{\delta}, a_{\delta}^{\text {int }}\right)$ is a directed half-edge pointing horizontally into $\Omega_{\delta}$, as in Figure 11. If $a_{\delta} \in \partial^{\mathrm{h}} \Omega_{\delta}^{\bullet}$, we let $a_{\delta}^{\text {int }}=a_{\delta}$ but sometimes interpret $a_{\delta}^{\text {int }}=a_{\delta} \pm 0 i$ as a point "just inside" $\Omega_{\delta}^{\bullet, \text { int }}$.

For $a_{\delta}$ as above and for a fixed $z \in \Omega_{\delta}^{\bullet}$, possibly $z=b_{\delta}$, we let $\psi=\psi_{a_{\delta}, z}^{\xi}: \Omega_{\delta}^{\bullet} \rightarrow\{0,1\}$ be a function satisfying the following:

(1) $\psi\left(a_{\delta}^{\text {int }}\right)=\psi(z)=1$ if $z \neq a_{\delta}^{\text {int }}$, respectively, $=0$ if $z=a_{\delta}^{\text {int }}$,

(2) $\psi(u)=0$ for all $u \in \partial^{\mathrm{h}} \Omega_{\delta}^{\bullet} \backslash\left\{a_{\delta}, z\right\}$,

(3) for $u \in \Omega_{\delta}^{\bullet, \text { int }}$ we have that $\psi(u+\varepsilon i)=1-\psi(u-\varepsilon i)$ for all small enough $\varepsilon>0$ if either $u \pm \delta / 2 \in \xi$ (that is, $u$ is an endpoint of a bridge) or $u \in\left(\left\{a_{\delta}^{\text {int }}\right\} \triangle\{z\}\right)$, and

(4) the set $I(\psi)=\left\{u \in \Omega_{\delta}^{\bullet}: \psi(u)=1\right\}$ is closed.

Thus $\psi$ is a random-parity configuration with sources $A=\left\{a_{\delta}^{\text {int }}\right\} \triangle\{z\}$ and boundary condition 0 on $\partial^{\mathrm{h}} \Omega_{\delta}^{\bullet}$. It is easy to see that there is at most one function $\psi_{a_{\delta}, z}^{\xi}$ satisfying the above constraints, for each given $\xi$ (and $\left.a_{\delta}, z\right)$. We let $\mathcal{A}\left(a_{\delta}, z\right)$ be the event (set of $\xi$ 's) such that there exists such a $\psi$. We also extend the definition of $\mathcal{A}\left(a_{\delta}, z\right)$ to allow $z \in \Omega_{\delta}^{\circ}$ by letting

$$
\mathcal{A}\left(a_{\delta}, z\right)=\mathcal{A}\left(a_{\delta}, z-\delta / 2\right) \cup \mathcal{A}\left(a_{\delta}, z+\delta / 2\right) \text { if } z \in \Omega_{\delta}^{\circ} .
$$

Note that this union is disjoint.

It is worth stating precisely a (necessary and sufficient) condition for $\xi$ to belong to $\mathcal{A}\left(a_{\delta}, z\right)$ when $z \in \Omega_{\delta}^{\bullet}$. To state the condition, let

$$
V(u)=\left\{u^{\prime} \in \Omega_{\delta}^{\bullet}:\left[u, u^{\prime}\right] \subseteq \Omega_{\delta}^{\bullet}\right\}, \text { for } u \in \Omega_{\delta}^{\bullet},
$$

be the maximal vertical line contained in $\Omega_{\delta}^{\bullet}$ and containing $u$. Let

$$
S_{a_{\delta}, z}^{\xi}(u)=\{v \in V(u): v \pm \delta / 2 \in \xi\} \cup\left(\left\{a_{\delta}^{\mathrm{int}}\right\} \triangle\{z\}\right)
$$

be the set of points in $V(u)$ where $\psi$ is required to change the value. Then, for $z \in \Omega_{\delta}^{\bullet}$,

$$
\xi \in \mathcal{A}\left(a_{\delta}, z\right) \Leftrightarrow\left|S_{a_{\delta}, z}^{\xi}(u)\right| \text { is even for all } u \in \Omega_{\delta}^{\bullet} .
$$

In words, $\psi$ must switch (from 0 to 1 or from 1 to 0 ) an even number of times on each line $V(u)$.
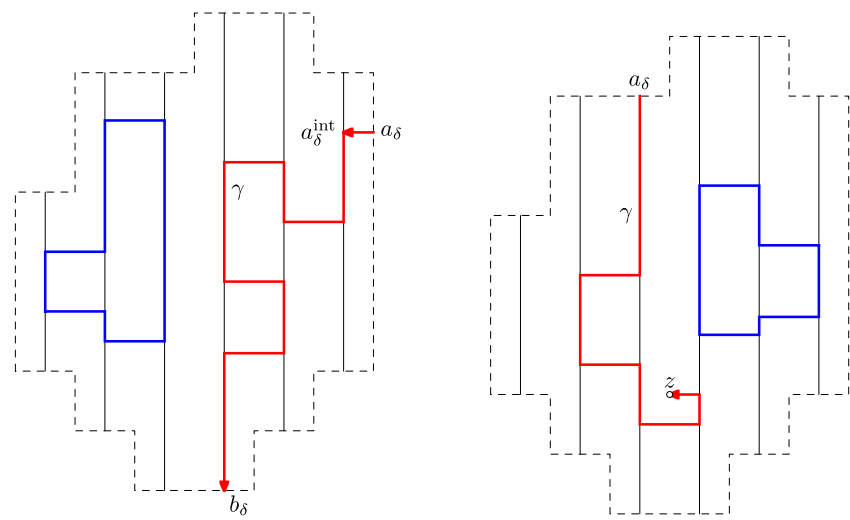

FIG. 11. Dual domain $\Omega_{\delta}$ with $\partial \Omega_{\delta}$ drawn dashed and $\Omega_{\delta}^{\bullet}$ drawn solid. Left: A labelling $\psi_{a_{\delta}, b_{\delta}}^{\xi}$, with points $u$ satisfying $\psi(u)=1$ marked fat, with blue for loops and red for the path $\gamma$. In this case $a_{\delta} \in \partial^{\mathrm{v}} \Omega_{\delta}^{\circ}$. Right: Same domain with a labelling $\psi_{a_{\delta}, z}^{\xi}$ for $z \in \Omega_{\delta}^{\circ, \text { int }}$. In this case $a_{\delta} \in \partial^{+} \Omega_{\delta}^{\bullet}$. 
If $u \in \Omega_{\delta}^{\bullet} \backslash\left\{a_{\delta}^{\text {int }}\right\}$ and $\xi \in \mathcal{A}\left(a_{\delta}, u\right)$, then $\psi=\psi_{a_{\delta}, u}^{\xi}$ contains a unique path $\gamma(\xi)$ from $a_{\delta}$ to $u$ which traverses the half-edge $\left(a_{\delta}, a_{\delta}^{\text {int }}\right)$ if $a_{\delta} \in \partial^{\mathrm{v}} \Omega_{\delta}^{\circ}$, intervals along which $\psi=1$, as well as bridges of $\xi$. For $w \in \Omega_{\delta}^{\circ}$ and $\xi \in \mathcal{A}\left(a_{\delta}, w\right)$, we complete $\gamma$ to form a path to $w$ by including the half-edge $(w-\delta / 2) \rightarrow w$ (if $\xi \in \mathcal{A}\left(a_{\delta}, w-\delta / 2\right)$ ), respectively, $w \leftarrow(w+\delta / 2)$ (if $\xi \in \mathcal{A}\left(a_{\delta}, w+\delta / 2\right)$ ). See Figure 11 . In the cases when $z \in\left\{a_{\delta}, a_{\delta}^{\text {int }}\right\}$ the path $\gamma$ is degenerate, and we interpret it as a small arrow (or half-edge) pointing from $a_{\delta}$ to $a_{\delta}^{\text {int }}$ if $z=a_{\delta}^{\text {int }}$, alternatively as a small path making an angle $\pi$ turn if $z=a_{\delta}$.

We define $W_{\gamma(\xi)}^{a_{\delta}, z}$ to be the winding-angle of $\gamma(\xi)$ from $a_{\delta}$ to $z$ (with $W^{a_{\delta}, a_{\delta}^{\text {int }}}=0$ and $W^{a_{\delta}, a_{\delta}}=\pi$ ). It is important to note that, in the case when $z=b_{\delta}$ is on the boundary, $W_{\gamma(\xi)}^{a_{\delta}, b_{\delta}}$ does not depend on $\xi$ (one cannot wind around the boundary, and $a_{\delta}, b_{\delta}$ have fixed orientations), i.e., it takes a fixed value which we denote $W^{a_{\delta}, b_{\delta}}$.

Write $1^{\circ}(z)$ for the indicator that $z \in \Omega_{\delta}^{\circ}$. Define the random variable

$$
X^{a_{\delta}, z}(\xi)=\mathbb{I}_{\mathcal{A}\left(a_{\delta}, z\right)}(\xi) \exp \left(-2 h\left|I\left(\psi_{a_{\delta}, z}^{\xi}\right)\right|\right)\left(\frac{1}{\sqrt{2}}\right)^{1^{\circ}(z)} .
$$

Definition 5.1. Write $\mathbb{E}=\mathbb{E}_{0,1 / 2 \delta}$ for the law of $\xi=\xi^{\circ}$ and let $\left(\Omega_{\delta}, a_{\delta}, b_{\delta}\right)$ be as above. Define the spin-observable

$$
F_{\delta}^{\mathrm{sp}}(z)=\frac{\mathbb{E}\left[\exp \left(-\frac{i}{2} W_{\gamma(\xi)}^{a_{\delta}, z}\right) X_{\gamma(\xi)}^{a_{\delta}, z}\right]}{\mathbb{E}\left[\exp \left(-\frac{i}{2} W_{\gamma(\xi)}^{a_{\delta}, b_{\delta}}\right) X_{\gamma(\xi)}^{a_{\delta}, b_{\delta}}\right]}, \quad z \in \Omega_{\delta}^{\bullet} \cup \Omega_{\delta}^{\circ} .
$$

Note that we have defined this observable using the random-parity representation, whose classical analogue is the random-current representation of Ref. 1 rather than the high-temperature expansion used by Chelkak and Smirnov. ${ }^{12}$ The high-temperature expansion is essentially the random-current representation "modulo two."

\section{B. S-holomorphicity}

In this section, we show the following result.

Theorem 5.2. Let $F_{\delta}^{\mathrm{sp}}$ be the spin-observable in a primal domain $\left(\Omega_{\delta}, a_{\delta}, b_{\delta}\right)$ with two marked points on the boundary, as above. Then $F_{\delta}^{\mathrm{sp}}$ is s-holomorphic at all $z \in\left(\Omega_{\delta}^{\bullet, \text { int }} \cup \Omega_{\delta}^{\circ \text {,int }}\right) \backslash\left\{a_{\delta}^{\mathrm{int}}, a_{\delta}^{\mathrm{int}} \pm \delta / 2\right\}$.

Regarding the behaviour near $a_{\delta}^{\text {int }}$, we note that half of condition (10) in Definition 3.1 holds at $a_{\delta}^{\text {int }}$, but condition (11) fails.

Since $a_{\delta}$ and $b_{\delta}$ are fixed, we will use the shorthands,

$$
\mathcal{W}^{z}(\xi)=W^{a_{\delta}, b_{\delta}}-W_{\gamma(\xi)}^{a_{\delta}, z}, \quad X^{z}(\xi)=X^{a_{\delta}, z}(\xi)
$$

Note that $F_{\delta}^{\mathrm{sp}}(z)$ is a real multiple of

$$
F_{\delta}(z)=\mathbb{E}\left[\exp \left(\frac{i}{2} \mathcal{W}^{z}(\xi)\right) X^{z}(\xi)\right],
$$

so it suffices to show s-holomorphicity of this $F_{\delta}(z)$.

It will be useful to note the following interpretation of the quantity $\mathcal{W}^{z}(\xi)$. Imagine that we augment $\gamma$ with a curve $\hat{\gamma}$ in $\Omega_{\delta}$ which starts at $z$ in the same direction that $\gamma$ ends, and which finishes at $b_{\delta}$ (pointing down). Let $W_{\hat{\gamma}}^{z, b_{\delta}}$ denote its winding angle. Then $\Gamma=\gamma \cup \hat{\gamma}$ is a curve in $\Omega_{\delta}^{\bullet}$ from $a_{\delta}$ to $b_{\delta}$, thus $\Gamma$ has a winding angle $W^{a_{\delta}, b_{\delta}}$, meaning that $\mathcal{W}^{z}(\xi)$ is the winding-angle from $z$ to $b_{\delta}$.

We now turn to the proof of Theorem 5.2. Recall that we define $F_{\delta}^{\alpha}(z)$ by

$$
F_{\delta}^{\alpha}(z)=\operatorname{Proj}\left[F_{\delta}(z) ; \ell(\alpha)\right], \quad \alpha \in\{\uparrow, \downarrow, \leftarrow, \rightarrow\} .
$$

This means that $F_{\delta}^{\alpha}$ automatically satisfy the relations of the $\Phi_{\delta}^{\alpha}$ in Lemma 4.3, that is,

$$
\begin{aligned}
& F_{\delta}^{\uparrow}(z)=\frac{1}{\sqrt{2}}\left(e^{i \pi / 4} F_{\delta}^{\leftarrow}(z)+e^{-i \pi / 4} F_{\delta}(z)\right), \\
& F_{\delta}^{\downarrow}(z)=\frac{1}{\sqrt{2}}\left(e^{i \pi / 4} F_{\delta}(z)+e^{-i \pi / 4} F_{\delta}^{\leftarrow}(z)\right) .
\end{aligned}
$$


If $z=u \in \Omega_{\delta}^{\bullet} \backslash\left\{a_{\delta}^{\text {int }}\right\}$, then $\gamma$ reaches $u$ either from below or from above; we write these events pictorially as

$$
\left\{\begin{array}{l}
u \\
\gamma
\end{array}\right\} \quad \text { and } \quad\left\{\begin{array}{l}
\gamma \\
\mathbf{v} u
\end{array}\right\}
$$

Similarly, if $z=w \in \Omega_{\delta}^{\circ}$, then $\gamma$ reaches $w$ either from the left or the right, pictorially represented as

$$
\{\underset{\infty}{w} \gamma\} \quad \text { and } \quad\left\{\begin{array}{ll}
\gamma & w \\
\hdashline 0
\end{array}\right\}
$$

Lemma 5.3. If $u \in \Omega_{\delta}^{\bullet} \backslash\left\{a_{\delta}^{\text {int }}\right\}$, then

$$
\begin{aligned}
& F_{\delta}^{\leftarrow}(u)=\mathbb{E}\left[\exp \left(\frac{i}{2} \mathcal{W}^{u}\right) X^{u} \mathbb{I}\left\{\begin{array}{l}
u \\
\gamma
\end{array}\right\}\right], \text { and } \\
& F_{\delta}(u)=\mathbb{E}\left[\exp \left(\frac{i}{2} \mathcal{W}^{u}\right) X^{u} \mathbb{I}\left\{\begin{array}{l}
\gamma \\
\gamma
\end{array}\right\}\right],
\end{aligned}
$$

and if $w \in \Omega_{\delta}^{\circ}$,

$$
\begin{aligned}
& F_{\delta}^{\uparrow}(w)=\mathbb{E}\left[\exp \left(\frac{i}{2} \mathcal{W}^{w}\right) X^{w} \mathbb{I}\left\{\begin{array}{ll}
\gamma & w \\
\underset{\infty}{0}
\end{array}\right\}\right], \text { and } \\
& F_{\delta}^{\downarrow}(w)=\mathbb{E}\left[\exp \left(\frac{i}{2} \mathcal{W}^{w}\right) X^{w} \mathbb{I}\left\{\begin{array}{ll}
w & \gamma \\
\propto &
\end{array}\right] .\right.
\end{aligned}
$$

Proof. We show (31) and the argument for (32) is similar. Certainly the two terms on the righthand-sides of (31) sum to $F_{\delta}(u)$. Moreover, if $\gamma$ reaches $u$ from below, then $\mathcal{W}^{u}=\pi+2 \pi n$ for some $n=n(\xi) \in \mathbb{Z}$, and if $\gamma$ reaches $u$ from above, then $\mathcal{W}^{u}=0+2 \pi n$ for some $n=n(\xi) \in \mathbb{Z}$. Thus the two terms belong to $\ell(\leftarrow)$ and $\ell(\rightarrow)$, respectively, and these two lines being perpendicular, the claim (31) follows.

Proposition 5.4. Conditions (9) and (10) in Definition 3.1 hold at all $z \in\left(\Omega_{\delta}^{\bullet, \text { int }} \cup \Omega_{\delta}^{\circ \text {,int }}\right) \backslash$ $\left\{a_{\delta}^{\text {int }}, a_{\delta}^{\text {int }} \pm \delta / 2\right\}$.

Proof. We give details for the case $z=w \in \Omega_{\delta}^{\circ \text {,int }}$, the case $z \in \Omega_{\delta}^{\bullet, \text { int }}$ being similar. Writing $u=w-\delta / 2, v=w+\delta / 2$, we need to show that (when neither $u$ nor $v$ equals $a_{\delta}^{\text {int }}$ )

$$
F_{\delta}^{\uparrow}(w)=F_{\delta}^{\uparrow}(u) \text { and } F_{\delta}^{\downarrow}(w)=F_{\delta}^{\downarrow}(v)
$$

We give details for the case $\uparrow$ only, the claim for $\downarrow$ again being similar.

Consider the terms in (31). Inside the expectations, we have

$$
\text { in } F_{\delta}^{\leftarrow}(u), \quad X^{u}(\xi)=\sqrt{2} X^{w}(\xi) \text { and } \mathcal{W}^{u}(\xi)=\mathcal{W}^{w}(\xi)-\frac{\pi}{2},
$$

since if we add the half-edge from $u$ to $w$, this puts an additional factor $1 / \sqrt{2}$ into $X$, and $\gamma$ does an additional $-\pi / 2$ turn. Similarly,

$$
\text { in } F_{\delta}(u), \quad X^{u}(\xi)=\sqrt{2} X^{w}(\xi) \text { and } \mathcal{W}^{u}(\xi)=\mathcal{W}^{w}(\xi)+\frac{\pi}{2}
$$

We use the symbolic notation

$$
\left\{\begin{array}{l}
\infty w \\
\gamma
\end{array}\right\} \quad \text { and } \quad\left\{\left\lfloor\begin{array}{l}
\gamma \\
\rightarrow \infty w
\end{array}\right\}\right.
$$


for the events that $\gamma$ ends with a right- or left-turn at $u$ into $w$, respectively. Using (30)-(34), we have for the case when neither $u$ nor $v$ equals $a_{\delta}^{\text {int }}$,

$$
\begin{aligned}
& F_{\delta}^{\uparrow}(u)=\frac{1}{\sqrt{2}}\left(e^{i \pi / 4} F_{\delta}^{\leftarrow}(u)+e^{-i \pi / 4} F_{\delta}(u)\right) \\
& =\frac{1}{\sqrt{2}} \mathbb{E}\left[\exp \left(\frac{i}{2}\left(\mathcal{W}^{u}+\frac{\pi}{2}\right)\right) X^{u} \mathbb{I}\left\{\begin{array}{l}
u \\
\gamma
\end{array}\right\}\right] \\
& +\frac{1}{\sqrt{2}} \mathbb{E}\left[\exp \left(\frac{i}{2}\left(\mathcal{W}^{u}-\frac{\pi}{2}\right)\right) X^{u} \mathbb{I}\left\{\begin{array}{l}
\gamma \\
u
\end{array}\right\}\right] \\
& =\mathbb{E}\left[\exp \left(\frac{i}{2} \mathcal{W}^{w}\right) X^{w} \mathbb{I}\left\{\left[\begin{array}{l}
{ }_{\gamma} w \\
\gamma
\end{array}\right\}\right]\right. \\
& +\mathbb{E}\left[\exp \left(\frac{i}{2} \mathcal{W}^{w}\right) X^{w} \mathbb{I}\left\{\left\lfloor_{\rightarrow \infty}^{\gamma}\right\}\right]\right. \\
& =\mathbb{E}\left[\exp \left(\frac{i}{2} \mathcal{W}^{w}\right) X^{w} \mathbb{I}\{\stackrel{\gamma}{\underset{\sim 0}{w}}\}\right] \\
& =F_{\delta}^{\uparrow}(w) \text {, as required. }
\end{aligned}
$$

The remaining conditions for s-holomorphicity take more work to verify. Theorem 5.2 follows once we establish the following.

Proposition 5.5. Condition (11) holds at all $z \in\left(\Omega_{\delta}^{\bullet, \text { int }} \cup \Omega_{\delta}^{\circ \text {,int }}\right) \backslash\left\{a_{\delta}^{\text {int }}, a_{\delta}^{\text {int }} \pm \delta / 2\right\}$.

Proof. Again we give details only for $z=w \in \Omega_{\delta}^{\circ \text {,int }}$. Writing $u=w-\delta / 2, v=w+\delta / 2$, we need to show (as long as neither $u$ nor $v$ equals $a_{\delta}^{\text {int }}$ ) that

$$
\dot{F}_{\delta}^{\uparrow}(w)=\frac{i}{\delta}\left(F_{\delta}^{\downarrow}(v)-F_{\delta}^{\downarrow}(u)\right) \text { and } \dot{F}_{\delta}^{\downarrow}(w)=\frac{i}{\delta}\left(F_{\delta}^{\uparrow}(v)-F_{\delta}^{\uparrow}(u)\right) .
$$

We give details only for the case of $\dot{F}_{\delta}^{\uparrow}(w)$. Take $\varepsilon>0$ small (the case $\varepsilon<0$ can be treated similarly), and consider $F_{\delta}^{\uparrow}(w+i \varepsilon)-F_{\delta}^{\uparrow}(w)$. Note from (32) that

$$
F_{\delta}^{\uparrow}(w)=\frac{1}{\sqrt{2}} \mathbb{E}\left[\exp \left(\frac{i}{2} \mathcal{W}^{w}\right) \exp \left(-2 h\left|I\left(\psi_{a_{\delta}, u}\right)\right|\right) \mathbb{I}_{\mathcal{A}\left(a_{\delta}, u\right)}\right] .
$$

Also note that $\mathcal{A}\left(a_{\delta}, u\right)=\mathcal{A}\left(a_{\delta}, u+i \varepsilon\right)$ as long as $[u, u+i \varepsilon] \subseteq \Omega_{\delta}^{\bullet}$. We may thus write

$$
\begin{aligned}
& F_{\delta}^{\uparrow}(w+i \varepsilon)-F_{\delta}^{\uparrow}(w) \\
& =\frac{1}{\sqrt{2}} \mathbb{E}\left[\left(e^{i / 2 \mathcal{W}^{w+i \varepsilon}} e^{-2 h\left|I\left(\psi_{a_{\delta}, u+i \varepsilon}\right)\right|}-e^{i / 2 \mathcal{W}^{w}} e^{-2 h\left|I\left(\psi_{a_{\delta}, u}\right)\right|}\right) \mathbb{I}_{\mathcal{A}\left(a_{\delta}, u\right)}\right] .
\end{aligned}
$$

We will split the expectation into the two cases: (i) $\xi(w, w+i \varepsilon)=0$ and (ii) $\xi(w, w+i \varepsilon)>0$, i.e., according to whether there is a bridge in the interval $(w, w+i \varepsilon)$ or not.

The first case, when there is no bridge, is illustrated in Figures 12 and 13. In this case, we have that $W_{\gamma}^{a_{\delta}, w+i \varepsilon}=W_{\gamma}^{a_{\delta}, w}$ and hence $\mathcal{W}^{w}=\mathcal{W}^{w+i \varepsilon}$. Let

$$
\hat{\varepsilon}=\left|I\left(\psi_{a_{\delta}, u}\right)\right|-\left|I\left(\psi_{a_{\delta}, u+i \varepsilon}\right)\right|,
$$

and note that $-\varepsilon \leq \hat{\varepsilon} \leq \varepsilon$. We may thus write the contribution from case (i) to the expectation in (35) as

$$
\frac{1}{\sqrt{2}} \mathbb{E}\left[e^{i / 2 \mathcal{W}^{w}} e^{-2 h\left|I\left(\psi_{a_{\delta}, u}\right)\right|}\left(e^{2 h \hat{\varepsilon}}-1\right) \mathbb{I}_{\mathcal{A}\left(a_{\delta}, u\right)} \mathbb{I}\{\xi(w, w+i \varepsilon)=0\}\right] .
$$

Since the factor $e^{2 h \hat{\varepsilon}}-1$ is of order $O(\varepsilon)$, we can (up to an error of order $O\left(\varepsilon^{2}\right)$ ) ignore events of probability $O(\varepsilon)$. Thus we may assume that there is no bridge in $(w-\delta, w-\delta+i \varepsilon)$ (i.e., we have a situation as in Figure 12, not as in Figure 13). Under the latter assumption, we have that

$$
\hat{\varepsilon}=\left\{\begin{array}{l}
+\varepsilon, \text { if } \gamma \text { comes from above } \\
-\varepsilon, \text { if } \gamma \text { comes from below }
\end{array}\right.
$$



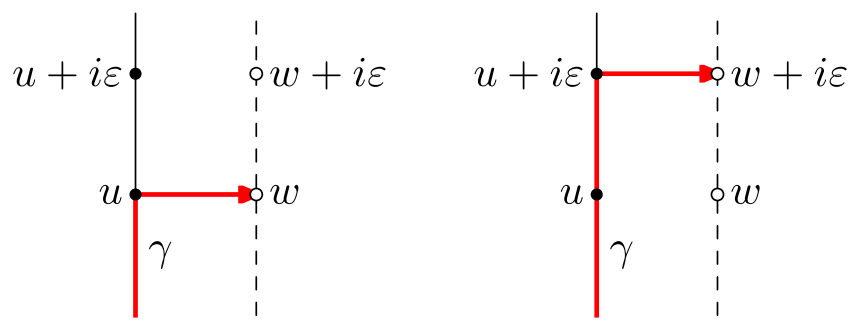

FIG. 12. The curve $\gamma$ finishes with a right turn at $u$ (in $\psi_{a, w}^{\xi}$, displayed left), respectively, $u+i \varepsilon$ (in $\psi_{a_{\delta}, w+i \varepsilon}^{\xi}$, displayed right). The winding angle is the same in both cases.
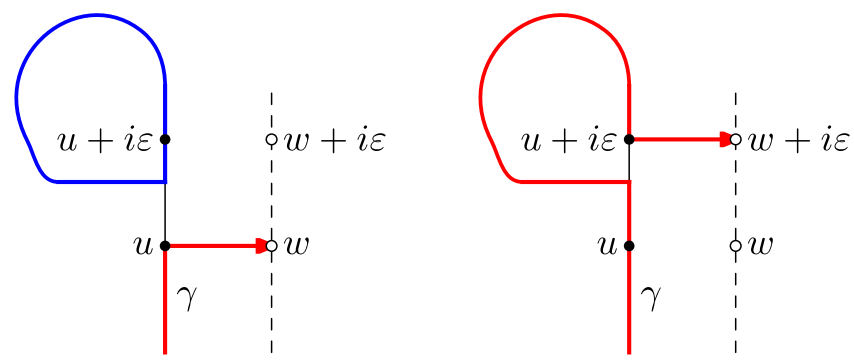

FIG. 13. Here $u+i \varepsilon$ is contained in a loop (in $\psi_{a_{\delta}, w}^{\xi}$, displayed left), which becomes part of $\gamma$ (in $\psi_{a_{\delta}, w+i \varepsilon}^{\xi}$, displayed right). The winding angle is still the same in both cases.

Thus, up to an error of order $O\left(\varepsilon^{2}\right)$, the integrand in (36) equals

$$
2 h \varepsilon\left(e^{i / 2 \mathcal{W}^{w}} e^{-2 h\left|I\left(\psi_{a_{\delta}, u}\right)\right|} \mathbb{I}\left\{\bigsqcup_{\rightarrow \infty}^{\gamma}\right\}\right\}-e^{i / 2 \mathcal{W}^{w}} e^{-2 h\left|I\left(\psi_{a_{\delta}, u}\right)\right|} \mathbb{I}\left\{\left\lceil{ }_{\gamma}^{\infty} w\right\}\right) .
$$

In the first term we have $\mathcal{W}^{w}=\mathcal{W}^{u}-\pi / 2$ and in the second term we have $\mathcal{W}^{w}=\mathcal{W}^{u}+\pi / 2$. Dividing by $\varepsilon$ and letting $\varepsilon \downarrow 0$, it follows that the contribution to $\dot{F}_{\delta}^{\uparrow}(w)$ from case (i) is

$$
\begin{aligned}
h & \sqrt{2}\left(e^{-i \pi / 4} F_{\delta}(u)-e^{i \pi / 4} F_{\delta}^{\leftarrow}(u)\right) \\
& =-i h \sqrt{2}\left(e^{i \pi / 4} F_{\delta}(u)+e^{-i \pi / 4} F_{\delta}^{\leftarrow}(u)\right) \\
& =-2 i h F_{\delta}^{\downarrow}(u)=-\frac{i}{\delta} F_{\delta}^{\downarrow}(u) .
\end{aligned}
$$

We now turn to case (ii), when there is a bridge in $(w, w+i \varepsilon)$. We need to show that the contribution from this case is $\frac{i}{\delta} F_{\delta}^{\downarrow}(w)=\frac{i}{\delta} F_{\delta}^{\downarrow}(v)$. We start by noting that, up to an error of order $O\left(\varepsilon^{2}\right)$, we may in fact assume that $\xi$ belongs to the event

$$
B=\left\{\begin{array}{l}
\xi(w, w+i \varepsilon)=1, \\
\xi(w-\delta, w-\delta+i \varepsilon)=0, \text { and } \\
\xi(w+\delta, w+\delta+i \varepsilon)=0 .
\end{array}\right\}
$$

The possible scenarios are illustrated in Figures 14-17. We write $\hat{w}$ for the location of the unique bridge in $(w, w+i \varepsilon)$. Recall the notation $\xi_{\hat{w}}=\xi \triangle\{\hat{w}\}$ for the configuration obtained by removing the bridge at $\hat{w}$ from $\xi$. We have that

$$
\xi \in \mathcal{A}\left(a_{\delta}, u\right) \Leftrightarrow \xi \in \mathcal{A}\left(a_{\delta}, u+i \varepsilon\right) \Leftrightarrow \xi_{\hat{w}} \in \mathcal{A}\left(a_{\delta}, v\right) .
$$

Moreover, the quantities

$$
\begin{aligned}
& \hat{\varepsilon}_{1}=\left|I\left(\psi_{a_{\delta}, v}^{\xi_{\hat{w}}}\right)\right|-\left|I\left(\psi_{a_{\delta}, u+i \varepsilon}^{\xi}\right)\right|, \\
& \hat{\varepsilon}_{2}=\left|I\left(\psi_{a_{\delta}, v}^{\xi} \hat{w}_{\hat{w}}\right)\right|-\left|I\left(\psi_{a_{\delta}, u}^{\xi}\right)\right|
\end{aligned}
$$



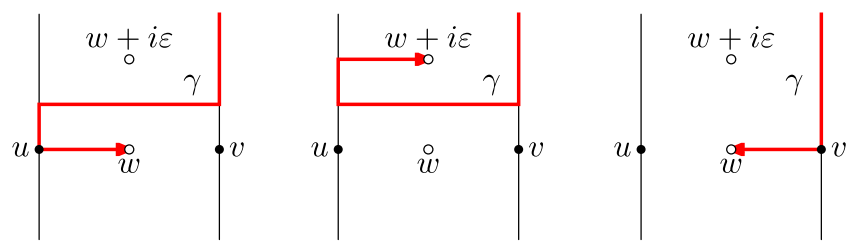

FIG. 14. Case (ii) (a), with $\psi_{a_{\delta}, w}^{\xi}$ to the left, $\psi_{a_{\delta}, w+i \varepsilon}^{\xi}$ in the middle, and $\psi_{a_{\delta}, w}^{\xi_{\hat{w}}}$ to the right.
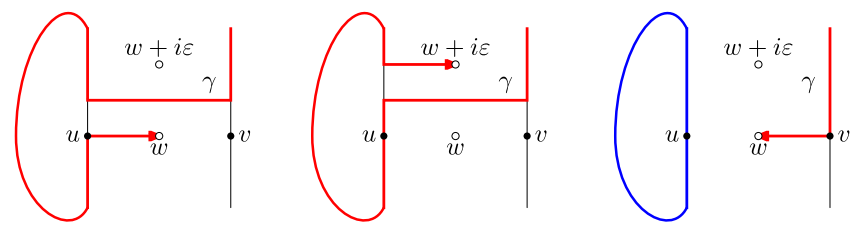

FIG. 15. Case (ii) (b), with $\psi_{a_{\delta}, w}^{\xi}$ to the left, $\psi_{a_{\delta}, w+i \varepsilon}^{\xi}$ in the middle, and $\psi_{a_{\delta}, w}^{\xi_{\hat{w}}}$ to the right.

satisfy $-2 \varepsilon \leq \hat{\varepsilon}_{1}, \hat{\varepsilon}_{2} \leq 2 \varepsilon$. The contribution from case (ii) to the expectation in (35) may thus, up to an error of order $O\left(\varepsilon^{2}\right)$, be written as

$$
\begin{aligned}
& \mathbb{E}\left[\mathbb{I}_{B}(\xi) \mathbb{I}_{\mathcal{A}\left(a_{\delta}, v\right)}\left(\xi_{\hat{w}}\right) X^{w}\left(\xi_{\hat{w}}\right)\left(e^{i / 2 \mathcal{W}^{w+i \varepsilon}(\xi)} e^{2 h \hat{\varepsilon}_{1}}-e^{i / 2 \mathcal{W}^{w}(\xi)} e^{2 h \hat{\varepsilon}_{2}}\right)\right] \\
& =\mathbb{E}\left[\mathbb{I}_{B}(\xi) \mathbb{I}_{\mathcal{A}\left(a_{\delta}, v\right)}\left(\xi_{\hat{w}}\right) X^{w}\left(\xi_{\hat{w}}\right)\left(e^{i / 2 \mathcal{W}^{w+i \varepsilon}(\xi)}-e^{i / 2 \mathcal{W}^{w}(\xi)}\right)\right]+O\left(\varepsilon^{2}\right) .
\end{aligned}
$$

We used that the event $B$ has probability $O(\varepsilon)$ and that both $e^{2 h \hat{\varepsilon}_{1}}=1+O(\varepsilon)$ and $e^{2 h \hat{\varepsilon}_{2}}=1+O(\varepsilon)$.

It remains to understand the factor

$$
\mathbb{I}_{B}(\xi) \mathbb{I}_{\mathcal{A}\left(a_{\delta}, v\right)}\left(\xi_{\hat{w}}\right)\left(e^{i / 2 \mathcal{W}^{w+i \varepsilon}(\xi)}-e^{i / 2 \mathcal{W}^{w}(\xi)}\right) .
$$

We claim that, for $\xi \in B$ and $\xi_{\hat{w}} \in \mathcal{A}\left(a_{\delta}, v\right)$,

$$
e^{i / 2 \mathcal{W}^{w+i \varepsilon}(\xi)}-e^{i / 2 \mathcal{W}^{w}(\xi)}=2 i \cdot e^{i / 2 \mathcal{W}^{w}\left(\xi_{\hat{w}}\right)} .
$$

Before showing this, we explain how to finish the proof. From (38), and assuming (39), the contribution to $\dot{F}_{\delta}^{\uparrow}(w)$ from case (ii) is

$$
\begin{aligned}
& 2 i \cdot \lim _{\varepsilon \downarrow 0} \frac{1}{\varepsilon} \mathbb{E}\left[\mathbb{I}_{B}(\xi) \mathbb{I}_{\mathcal{A}\left(a_{\delta}, v\right)}\left(\xi_{\hat{w}}\right) X^{w}\left(\xi_{\hat{w}}\right) e^{i / 2 \mathcal{W}^{w}\left(\xi_{\hat{w}}\right)}\right] \\
& =2 i J \mathbb{E}\left[e^{i / 2 \mathcal{W}^{w}(\xi)} X^{w}(\xi) \mathbb{I}_{\mathcal{A}\left(a_{\delta}, v\right)}(\xi)\right] \\
& =\frac{i}{\delta} F_{\delta}^{\downarrow}(w),
\end{aligned}
$$

as required. (We used (32) and the fact that the conditional distribution of $\xi_{\hat{w}}$ given $B$ converges to that of $\xi$ as $\varepsilon \rightarrow 0$.)

It remains to show (39). There are 4 sub-cases to consider, depending on whether $\gamma$ traverses $\hat{w}$ (in $\psi_{a_{\delta}, u}^{\xi}$ ), in which direction, etc. The first case, which we call case (a), is defined by the condition
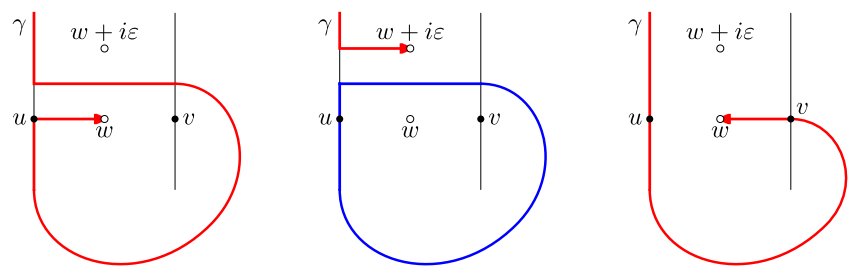

FIG. 16. Case (ii) (c), with $\psi_{a_{\delta}, w}^{\xi}$ to the left, $\psi_{a_{\delta}, w+i \varepsilon}^{\xi}$ in the middle, and $\psi_{a_{\delta}, w}^{\xi_{\hat{w}}}$ to the right. 

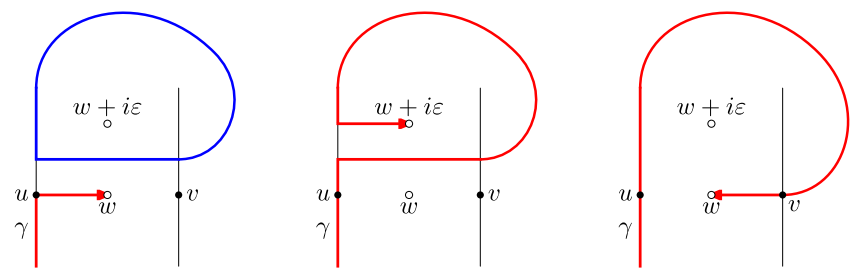

FIG. 17. Case (ii) (d), with $\psi_{a_{\delta}, w}^{\xi}$ to the left, $\psi_{a_{\delta}, w+i \varepsilon}^{\xi}$ in the middle, and $\psi_{a_{\delta}, w}^{\xi_{\hat{w}}}$ to the right.

$\psi_{a_{\delta}, u}^{\xi}(u+0 i)=1$ and is depicted in Figure 14 . In this case $\gamma(\xi)$ necessarily traverses $\hat{w}$ from right to left.

It is not hard to see that we get

$$
\text { case (a): } \quad \mathcal{W}^{w}(\xi)=\mathcal{W}^{w}\left(\xi_{\hat{w}}\right)-\pi, \quad \mathcal{W}^{w+i \varepsilon}(\xi)=\mathcal{W}^{w}\left(\xi_{\hat{w}}\right)+\pi .
$$

This establishes (39) for case (a). In the remaining 3 cases, we will have $\psi_{a_{\delta}, u}^{\xi}(u+0 i)=0$, meaning that (in $\left.\psi_{a_{\delta}, u}^{\xi}\right) \gamma$ can traverse $\hat{w}$ from right to left (case (b)), from left to right (case (c)), or not at all (case (d)). The cases are depicted in Figures 15-17, respectively. We get the following:

$$
\begin{array}{lll}
\text { case (b): } & \mathcal{W}^{w}(\xi)=\mathcal{W}^{w}\left(\xi_{\hat{w}}\right)-\pi, & \mathcal{W}^{w+i \varepsilon}(\xi)=\mathcal{W}^{w}\left(\xi_{\hat{w}}\right)+\pi \\
\text { case (c): } & \mathcal{W}^{w}(\xi)=\mathcal{W}^{w}\left(\xi_{\hat{w}}\right)+3 \pi, & \mathcal{W}^{w+i \varepsilon}(\xi)=\mathcal{W}^{w}\left(\xi_{\hat{w}}\right)+\pi \\
\text { case (d): } & \mathcal{W}^{w}(\xi)=\mathcal{W}^{w}\left(\xi_{\hat{w}}\right)-\pi, & \mathcal{W}^{w+i \varepsilon}(\xi)=\mathcal{W}^{w}\left(\xi_{\hat{w}}\right)-3 \pi
\end{array}
$$

In all cases, we see that (39) holds, as claimed.

\section{DISCUSSION}

\section{A. Convergence of the observables}

As mentioned in the Introduction, we expect that both the FK- and spin-observables, suitably rescaled, converge as $\delta \rightarrow 0$. We sketch an outline of a possible argument, following the arguments for the classical case (see Refs. 12,15, and 24). As also mentioned, the details in the case of the FKobservable were supplied by $\mathrm{Li}^{20}$ shortly after this paper was finished. We take the discrete domains $\left(\Omega_{\delta}, a_{\delta}, b_{\delta}\right)$ to approximate a continuous domain $(\Omega, a, b)$ (e.g., in the Carathéodory sense, i.e., convergence on compact subsets of suitably normalized conformal maps from the upper half-plane into the domains, see Ref. 15, Definition 3.10).

The two main steps are to show (i) precompactness of sequences of s-holomorphic functions $\left(F_{\delta}\right)_{\delta>0}$ and (ii) convergence of the auxiliary functions $\left(H_{\delta}\right)_{\delta>0}$ given in Proposition 3.2.

For (i), note first that preholomorphic functions, and hence in particular s-holomorphic functions, are $\Delta_{\delta}$-harmonic. Indeed, if $F_{\delta}$ satisfies (8) at $z$ and $z \pm \delta / 2$, then differentiating twice using (8) gives

$$
\begin{aligned}
\ddot{F}_{\delta}(z) & =\frac{1}{i \delta}\left(\dot{F}_{\delta}(z-\delta / 2)-\dot{F}_{\delta}(z+\delta / 2)\right) \\
& =-\frac{1}{\delta^{2}}\left(F_{\delta}(z-\delta)+F_{\delta}(z+\delta)-2 F_{\delta}(z)\right) .
\end{aligned}
$$

Thus precompactness of s-holomorphic functions would follow from Lipschitzness of $\Delta_{\delta}$-harmonic functions combined with a suitable boundedness condition, using the Arzela-Ascoli theorem as in Ref. 15, Proposition 8.7. Completing this argument would require estimates for the Green's function $G_{\delta}(\cdot)$ in $\mathbb{C}_{\delta}^{\bullet}$, in particular, a suitable form of the asymptotics of $G_{\delta}(z)$ as $|z| \rightarrow \infty$ as in Refs. 19, 9, and 13. See Section III D of Li's paper ${ }^{20}$ for details in the present context.

For (ii), consider the sub- and superharmonic functions $H_{\delta}^{\bullet}=\left.H_{\delta}\right|_{\Omega_{\delta}^{\circ}}$ and $H_{\delta}^{\circ}=\left.H_{\delta}\right|_{\Omega_{\delta}^{\circ}}$ (see Proposition 3.2). It is not hard to partly determine the behaviour of these functions on the boundary. In the case of the FK-observable, we can choose the additive constant so that $H_{\delta}^{\bullet}=1$ on the black part $\partial_{\delta}^{\bullet}$ and $H_{\delta}^{\circ}=0$ on the white part $\partial_{\delta}^{\circ}$. In the case of the spin-observable, the constant can be chosen so that $H_{\delta}^{\circ}(w)=0$ for all $w \in \partial \Omega_{\delta}^{\circ} \backslash\left\{a_{\delta}\right\}$ (note also that $v(z)^{1 / 2} F_{\delta}^{\mathrm{sp}}(z) \in \mathbb{R}$ for all $z \in \partial^{\mathrm{v}} \Omega_{\delta}^{\circ} \cup \partial^{\mathrm{h}} \Omega_{\delta}^{\bullet}$ where $v(z)$ is the counter-clockwise oriented unit tangent). 
To fully determine the boundary-behaviour, one could try to use a variant of the "boundary modification trick" of Ref. 12 (this is the approach taken by $\mathrm{Li}^{20}$ ). In the case of the FK-observable, one could alternatively note that the difference of $H_{\delta}$ on the boundary and "just inside" the boundary is proportional to a percolation-probability which converges to zero away from $a_{\delta}, b_{\delta}$, like in the original argument for the square-lattice case $^{24}$ (this uses that the phase-transition is continuous ${ }^{8}$ ). Having determined the boundary-values of $H_{\delta}^{\bullet}$ and $H_{\delta}^{\circ}$, one would show that these functions are close to the harmonic function $h$ in $\Omega$ with the corresponding boundary-values. In the case of the FK-observable, we have $h=1$ on the clockwise arc from $a$ to $b$ and $h=0$ on the counter-clockwise arc, whereas for the spin-observable, we have $h=0$ on $\partial \Omega \backslash\{a\}$.

Since these are also the boundary-conditions for the classical case, ${ }^{12,24}$ we expect the observables to converge to the same limits under the same rescaling, namely,

$$
\frac{1}{\sqrt{\delta}} F_{\delta}^{\mathrm{FK}}(\cdot) \rightarrow \sqrt{\phi^{\prime}(\cdot)}, \quad F_{\delta}^{\mathrm{sp}}(\cdot) \rightarrow \sqrt{\frac{\psi^{\prime}(\cdot)}{\psi^{\prime}(b)}},
$$

where $\phi$ is a conformal map from $\Omega$ to $\mathbb{R}+i(0,1)$ mapping $a$ to $-\infty$ and $b$ to $+\infty$, and $\psi$ is a conformal map from $\Omega$ to the upper half-plane mapping $a$ to $\infty$ and $b$ to 0 . As mentioned, the first of these limits has now been established by $\mathrm{Li}^{20}$

\section{B. Parafermionic observables}

Recall from (17) that the FK-Ising model at the critical parameters $h=J=1 / 2 \delta$ has density proportional to $(\sqrt{2})^{L(\xi)}$ with respect to a Poisson law, where $L(\xi)$ is the number of loops. It is natural to ask also about measures with density $(\sqrt{q})^{L(\xi)}$ for other $q>0$. Such measures arise in the Aizenman-Nachtergaele representation ${ }^{3}$ of a class of quantum spin systems which includes the (spin- $\frac{1}{2}$ ) Heisenberg anti-ferromagnet as the case $q=4$. One may define an analog of the FK-Ising observable (20) which is also a direct analog of Smirnov's parafermionic observable for critical random-cluster models. ${ }^{24}$ We briefly describe this now.

Let $\left(\Omega_{\delta}, a_{\delta}, b_{\delta}\right)$ be a Dobrushin-domain as in Section IV and let $\sigma$ satisfy $\sin \left(\sigma \frac{\pi}{2}\right)=\frac{1}{2} \sqrt{q}$. Thus $\sigma=\frac{1}{2}$ for $q=2$ (TFIM) and $\sigma=1$ for $q=4$ (Heisenberg model). Recall the events $\Gamma_{z}^{\alpha}=\{\gamma$ passes $z$ in direction $\alpha\}$ and the winding-angle $W_{\gamma}^{\alpha}(z)$ of the interface to the exit. We now define

$$
\varphi^{\alpha}(\xi ; z)=\mathbb{I}_{\Gamma_{z}^{\alpha}}(\xi) \exp \left(i \sigma W_{\gamma(\xi)}^{\alpha}(z)\right) .
$$

Let $\hat{\mathbb{E}}_{\delta}$ denote the measure with density proportional to $(\sqrt{q})^{L(\xi)}$ with respect to the Poisson law with rate $\frac{1}{\delta \sqrt{q}}$. Similarly to before we define observables

$$
\Phi_{\delta}^{\uparrow}(z)=\hat{\mathbb{E}}_{\delta}\left[\varphi^{\uparrow}(\xi ; z)\right], \quad \Phi_{\delta}^{\downarrow}(z)=\hat{\mathbb{E}}_{\delta}\left[\varphi^{\downarrow}(\xi ; z)\right],
$$

as well as $F_{\delta}(z)=\Phi_{\delta}^{\uparrow}(z)+\Phi_{\delta}^{\downarrow}(z)$. Some properties of these quantities are immediate, e.g., for $w$ $\in \Omega_{\delta}^{\circ \text {,int }}$ we still have $\Phi_{\delta}^{\uparrow}(w)=\Phi_{\delta}^{\uparrow}(w-\delta / 2)$ and $\Phi_{\delta}^{\downarrow}(w)=\Phi_{\delta}^{\downarrow}(w+\delta / 2)$. Also, an adjusted version of Lemma 4.4 holds, with the factors $\frac{1}{\delta \sqrt{2}}$ replaced by $\frac{1}{\delta \sqrt{q}}$ and $e^{ \pm i \pi / 4}$ replaced by $e^{ \pm i \sigma \pi / 2}$. It might be interesting to investigate these observables further, especially due to the connection with the Heisenberg antiferromagnet.

\section{ACKNOWLEDGMENTS}

This work was mainly carried out while the author was at the University of Copenhagen in Denmark. The author is now supported by Vetenskapsrådet Grant No. 2015-05195. We would like to thank an anonymous referee for carefully reading the manuscript and helpfully suggesting improvements to the presentation.

\footnotetext{
${ }^{1}$ Aizenman, M., "Geometric analysis of $\phi^{4}$ fields and Ising models. Parts I and II," Commun. Math. Phys. 86, 1-48 (1982).

2 Aizenman, M., Klein, A., and Newman, C. M., "Percolation methods for dis-ordered quantum Ising models," in Phase Transitions: Mathematics, Physics, Biology, edited by R. Kotecký (World Scientific, Singapore, 1992).

${ }^{3}$ Aizenman, M. and Nachtergaele, B., "Geometric aspects of quantum spin states," Commun. Math. Phys. 164, 17-63 (1994)
} 
${ }^{4}$ Benoist, S. and Hongler, C., "The scaling limit of critical Ising interfaces is CLE(3)," Annals of Probability (to be published); e-print arXiv:1604.06975 (2016).

${ }^{5}$ Björnberg, J. E., "Graphical representations of Ising and Potts models," Ph.D. thesis, University of Cambridge, 2009, available at e-print arXiv:1011.2683.

${ }^{6}$ Björnberg, J. E., "Infrared bound and mean-field behaviour in the quantum Ising model," Commun. Math. Phys. 323(2), 329-366 (2013).

${ }^{7}$ Björnberg, J. E., "Vanishing critical magnetization in the quantum Ising model," Commun. Math. Phys. 337(2), 879-907 (2015).

${ }^{8}$ Björnberg, J. E. and Grimmett, G. R., “The phase transition of the quantum Ising model is sharp,” J. Stat. Phys. 136(2), 231 (2009).

${ }^{9}$ Bücking, U., "Approximation of conformal mappings by circle patterns,” Geom. Dedicata 137, 163-197 (2008).

${ }^{10}$ Chelkak, D., Duminil-Copin, H., Hongler, C., Kemppainen, A., and Smirnov, S., "Convergence of Ising interfaces to Schramm's SLE curves," C. R. Math. 352(2), 157-161 (2014).

${ }^{11}$ Chelkak, D., Hongler, C., and Izyurov, K., "Conformal invariance of spin correlations in the planar Ising model," Ann. Math. 181, 1087-1138 (2015).

${ }^{12}$ Chelkak, D. and Smirnov, S., "Universality in the 2D Ising model and conformal invariance of fermionic observables," Invent. Math. 189(3), 515-580 (2012).

${ }^{13}$ Chelkak, D. and Smirnov, S., "Discrete complex analysis on isoradial graphs," Adv. Math. 228(3), 1590-1630 (2011).

${ }^{14}$ Daley, D. J. and Vere-Jones, D., An Introduction to the Theory of Point Processes: Volume 2: General Theory and Structure (Springer, 2007).

${ }^{15}$ Duminil-Copin, H., "Parafermionic observables and their applications to planar statistical physics models," Ensaios Matematicos 25, 1-371 (2013)

${ }^{16}$ Hongler, C. and Smirnov, S., "The energy density in the planar Ising model," Acta Math. 211(2), 191-225 (2013).

${ }^{17}$ Ioffe, D., "Stochastic geometry of classical and quantum Ising models," in Methods of Contemporary Mathematical Statistical Physics, Volume 1970 of Lecture Notes in Mathematics (Springer, Berlin, 2009).

${ }^{18}$ Kemppainen, A. and Smirnov, S., "Conformal invariance of boundary touching loops of FK Ising model," e-print arXiv:1509.08858 (2015).

${ }^{19}$ Kenyon, R., "The Laplacian and Dirac operators on critical planar graphs," Invent. Math. 150, 409-439 (2002).

${ }^{20} \mathrm{Li}$, J.-H., "The interface of the FK-representation of the quantum Ising model converges to the SLE $16 / 3$," e-print arXiv:1608.02821 (2016).

${ }^{21}$ Lieb, E., Schultz, T., and Mattis, D., "Two soluble models of an antiferromagnetic chain,” Ann. Phys. 16, $407-466$ (1961).

${ }^{22}$ Pfeuty, P., "The one-dimensional Ising model with a transverse field," Ann. Phys. 57(1), 79-90 (1970).

${ }^{23}$ Smirnov, S., "Towards conformal invariance of 2D lattice models," in Proceedings of the International Congress of Mathematicians (European Mathematical Society, 2006), Vol. II, pp. 1421-1451.

${ }^{24}$ Smirnov, S., "Conformal invariance in random cluster models. I. Holomorphic fermions in the Ising model," Ann. Math. 172(2), 1435-1467 (2010). 